\title{
About the Memory of Transformation-Induced Plasticity in 35NCD16 Carbon Steel Subjected to Various Thermomechanical Histories
}

\author{
Jose Jimenez *(D) and Lakhdar Taleb
}

check for updates

Citation: Jimenez, J.; Taleb, L. About the Memory of TransformationInduced Plasticity in 35NCD16 Carbon Steel Subjected to Various Thermomechanical Histories. Metals 2021, 11, 1929. https://doi.org/ $10.3390 /$ met11121929

Academic Editor: Daixiu Wei

Received: 20 October 2021

Accepted: 25 November 2021

Published: 29 November 2021

Publisher's Note: MDPI stays neutral with regard to jurisdictional claims in published maps and institutional affiliations.

Copyright: (c) 2021 by the authors. Licensee MDPI, Basel, Switzerland. This article is an open access article distributed under the terms and conditions of the Creative Commons Attribution (CC BY) license (https:/ / creativecommons.org/licenses/by/ $4.0 /)$.
Materials Physics Group (GPM), Mixed Research Unit for the French National Centre for Scientific Research (UMR CNRS) UMR CNRS 6634, National Institute of Applied Sciences (INSA) of Rouen, 76800 St Etienne du Rouvray, France; lakhdar.taleb@insa-rouen.fr

* Correspondence: jose.jimenez_reyes@insa-rouen.fr

\begin{abstract}
This study deals with Transformation-Induced Plasticity (TRIP) observed in the martensitic transformation of 35NCD16 ferritic steel. In this study, TRIP tests were carried out for two different cases: First, after only free dilatometric (FD) tests, which is used as the reference test for the considered applied stress; second, with TRIP tests being performed similarly to the first case (same thermal cycle, same applied stress) but with pre-thermomechanical loading histories applied. Such histories may be FD tests, TRIP tests, elastoplastic history, etc. The comparison between the results of TRIP test (a) and TRIP test (b) indicates if TRIP holds the memory of the applied loading histories. The current obtained results tell us that TRIP does not hold any significant memory. During the martensite $\rightarrow$ austenite transformation, the material may present recovery from strain hardening. Waiting for more details about the physical phenomena responsible for the absence of TRIP memory, one can point out the importance of this result as it enables one to use the same specimen for several TRIP tests. However, this result must be validated using other combinations of loading histories (such as multiaxial and cyclic, among others).
\end{abstract}

Keywords: TRIP (transformation-induced plasticity); FD (free dilatometric) test; martensite; austenite; elastoplasticity; 35NCD16 ferritic steel

\section{Introduction}

Transformation-induced plasticity (TRIP) refers to an anomalous plastic strain observed when metallurgical transformation occurs under external stress much lower than the yield limit [1-4]. One of the most common ways of studying the TRIP phenomena is by performing a TRIP test. However, before defining this, we refer first to the definition of a free dilatometric (FD) test.

FD tests consist of subjecting a test specimen to a thermal cycle, which leads to the transformation of the $\alpha$ (ferritic) phase into the $\gamma$ (austenitic) phase during heating, followed by the transformation of the $\gamma$ phase into the $\alpha$ phase during cooling. During FD tests, the test specimen is only subjected to temperature variation and no inelastic strain is observed at the end of the transformation [3-5].

Similar to FD tests, TRIP tests also consist of subjecting a specimen to a thermal cycle leading to the same transformations just mentioned. However, the key difference between the FD tests and the TRIP tests is that during the TRIP tests, the test specimen is also subjected to the aforementioned external stress, provoking said anomalous plastic strain. The external stress is applied just before the starting temperature (Ms) of the transformation during cooling and maintained constant during the whole transformation [4]. 
The explanation of the TRIP phenomena dates back all the way to the 1960s, with two main mechanisms:

- Greenwood-Johnson mechanism: TRIP is due to the micro-mechanical plastic strain arising in the parent phase from the expansion of the product phase [6].

- Magee mechanism: TRIP is due to the formation of selected martensitic variants resulting from the applied stress [7].

Following these works there have been many contributions towards the understanding of the TRIP phenomena throughout the years, both from experimental and modeling point of views. Experimentally, one can cite the recent investigations of Otsuka et al. (2018) [8,9] about the effect of carbon amount on anisotropic dilatation behavior during phase transformation, in addition to the studies of Boudiaf (2011) [5] about the influence of the grain size of the austenitic phase on the TRIP and the recent study of Simsir (2017) [10] about the TRIP in martensitic and bainitic transformations of SAE52100 steel. Other works performed by Nagayama et al. (2000, 2001, 2002) [11-13] considering the martensitic transformation of Cr-Ni-Mo-Al-Ti steel show the existence of an interaction between classical plasticity and TRIP. For the modeling point of view, among the works proposed in the literature, one can cite the finite elements (FE) investigations of Tahimi (2011) [3] and, the works of Zuo et al. (2020) [14] on 40CrNiMo steel leading to a modification of the function that describes the kinetics of the TRIP. However, the well-known Leblond's model (1989) [15] seems the most important achievement. This model has been revised in Taleb and Sidoroff (2003) [1].

The focus of this paper is to provide our own small contribution to this subject, by carrying out experiments using TRIP and FD tests and analyzing the behavior of 35NCD15 ferritic steel test samples, and determining whether, within the experimental results, there is any observable elastoplastic history effect carried forward after different tests.

\section{Experimental Procedure}

In addition to the FD and TRIP tests, other tests have been carried out in order to gain an idea about the elastoplastic behavior of the austenitic phase at a given temperature before Ms (austenite $\rightarrow$ martensite transformation). Such elastoplastic characterization is performed through the application of a loading-unloading cycle where both directions are considered: Tension and torsion. The FD, TRIP, and elastoplastic tests are carried out in different orders (and magnitudes of stress in the case of the TRIP test) and are compared in order to visualize the transformation plasticity phenomenon and see if the effects of loading history could be observed.

As a remark, some details about the parameters are mentioned in Table 1.

Table 1. Different histories applied in the present work.

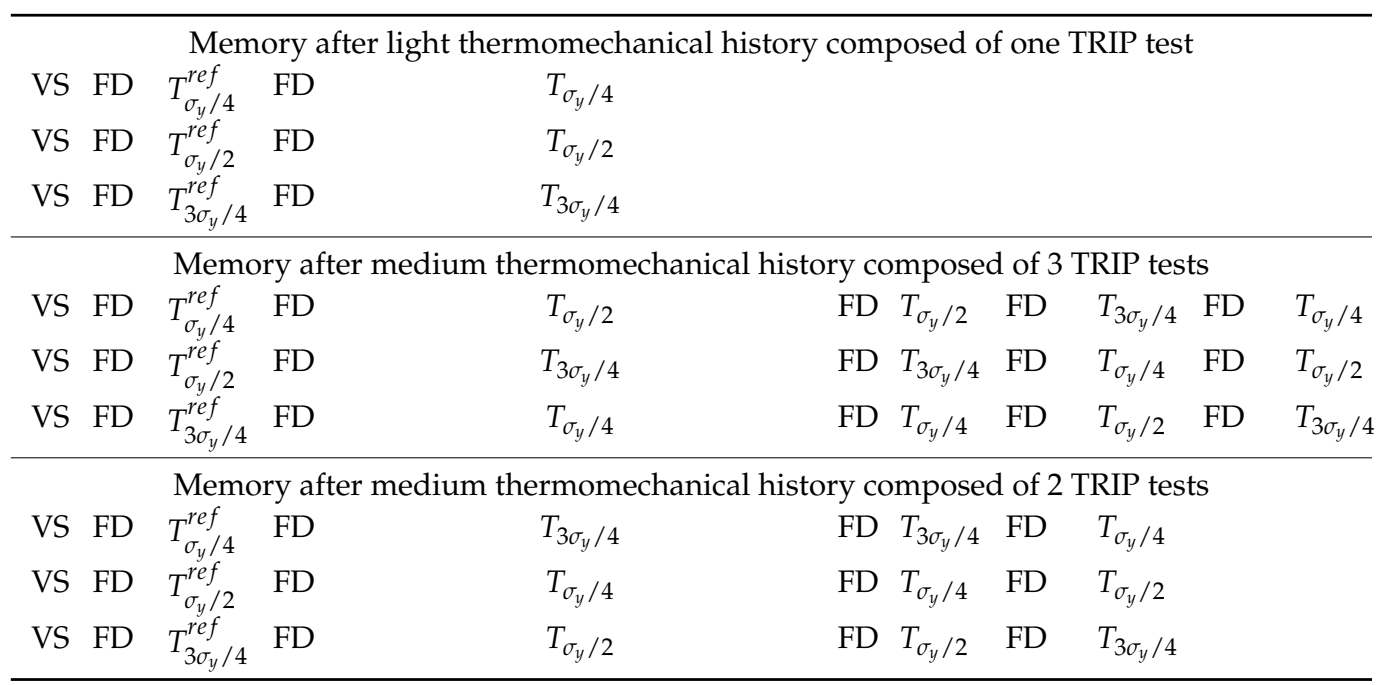


Table 1. Cont.

\begin{tabular}{lllllll}
\hline \multicolumn{6}{c}{ Memory after severe thermomechanical history in classical elastoplasticity } \\
VS & FD & $T_{\sigma_{y} / 4}^{r e f}$ & FD & Elastoplastic cycle (see Figure 4) & FD & $T_{\sigma_{y} / 4}$ \\
VS & FD & $T_{\sigma_{y} / 2}^{r e f}$ & FD & Elastoplastic cycle (see Figure 4) & FD & $T_{\sigma_{y} / 2}$ \\
VS & FD & $T_{3 \sigma_{y} / 4}^{r e f}$ & FD & Elastoplastic cycle (see Figure 4) & FD & $T_{3 \sigma_{y} / 4}$ \\
\hline
\end{tabular}

where:

- VS: Virgin specimen.

- $\quad T_{\sigma_{y} / 4}:$ TRIP test under $\sigma_{y} / 4$.

- $\quad T_{\sigma_{y} / 4}^{r e f}$ : reference TRIP test under $\sigma_{y} / 4$ (this test is considered as a reference, as it is preceded only by a free dilatometric cycle and will be used for comparisons throughout the article).

The $T_{\sigma_{y} / 4}$ test results will be compared to $T_{\sigma_{y} / 4}^{r e f}$ and, more generally, the test results of the third column will be compared to the corresponding test results of the last column.

The tests were carried out following the left to right order. An example for the first line of Table 1 is shown, in which the virgin specimen is subjected to successively:

FD test $\rightarrow$ TRIP test under $\sigma_{y} / 4 \rightarrow$ FD test $\rightarrow$ TRIP test under $\sigma_{y} / 4$.

In Figure 1, we can observe an example of how these tests are carried out by presenting a simplified form of the loading vs. time plot of the first series of tests previously presented.

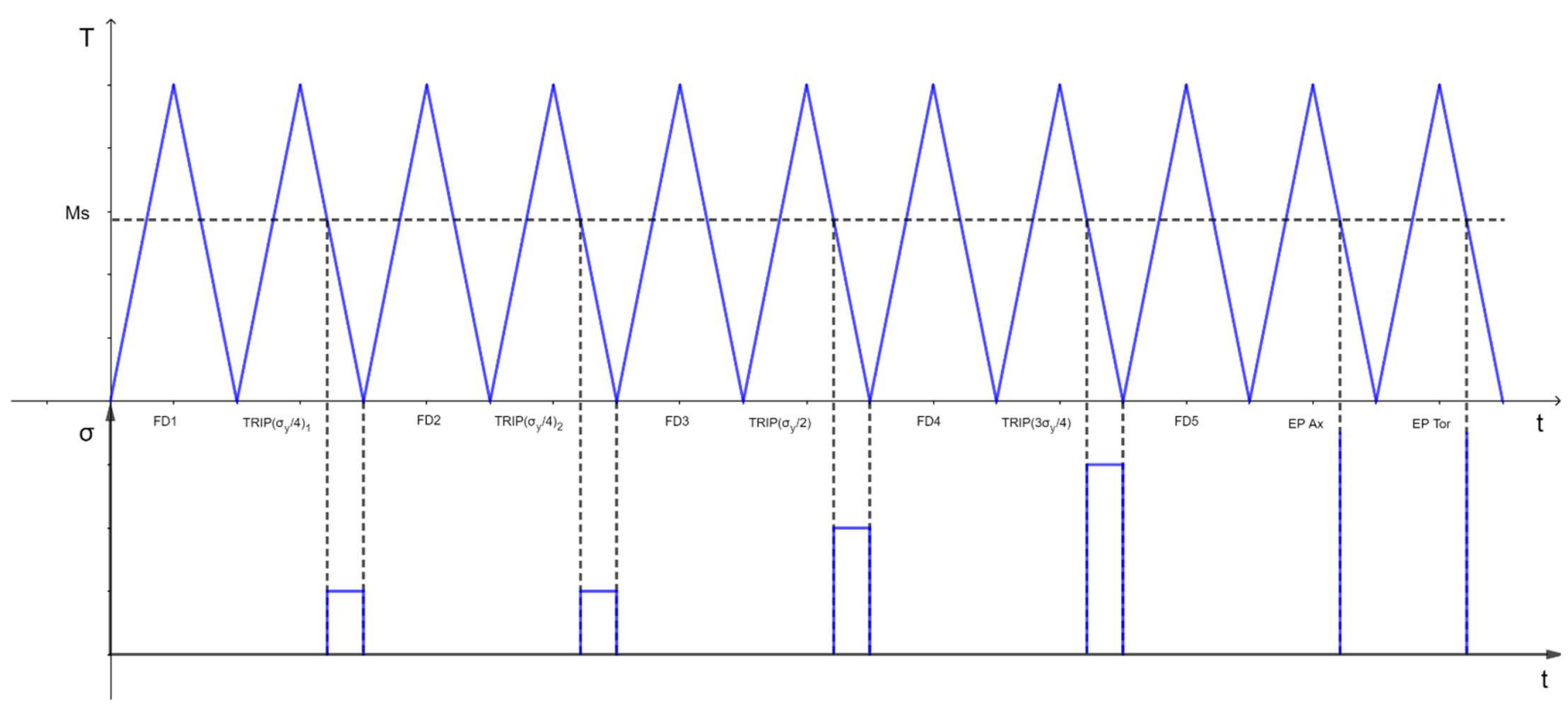

Figure 1. Simplified look of the loading vs. time plot of the first series of tests.

The top part of this figure shows an example of the temperature cycles through which the test specimen goes through. The Ms line represents the temperature at which the transformations would occur. The captions indicate the test cycles carried out. The stress charges are represented on the bottom part of the figure.

\subsection{Experimental Device}

In order to carry out the experiments, the device, which is a Series 809 Axial/Torsional Test System (MTS, Minneapolis, MN, USA) presented in Figure 2 is used. It is composed of 2 main parts: The part for thermal loading, and the part for mechanical loading.

The thermal loading is performed by electromagnetic induction, which is convenient for controlling a fast temperature rise. As for the cooling, this is performed using nitrogen and argon. The nitrogen flows inside the test specimen, while the argon flows on the 
external surface. The temperature measurements are made with K-type thermocouples, which are welded on the strain gage of the specimen.

The mechanical loading is conducted through a hydraulic multiaxial thermomechanical testing machine capable of working with axial, torsion, and internal pressure. The measurement of the elongation and rotation of the working zone is performed with a high-temperature bi-axial extensometer.

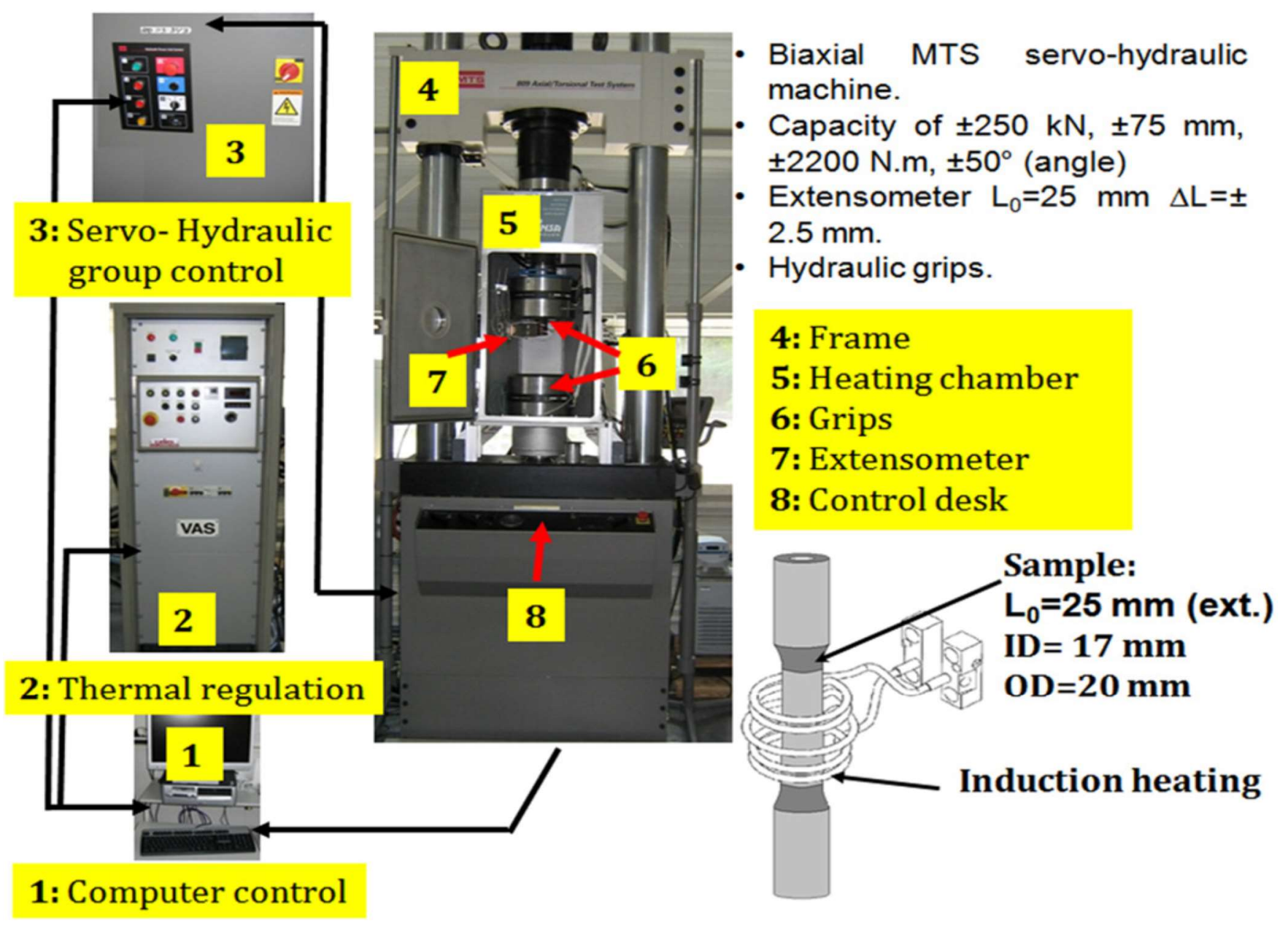

Figure 2. Experimental device used in the present study.

\subsection{Material and Specimen}

The chemical composition of the 35NCD16 steel test specimen used in this work is listed in Table 2. It is low-alloyed steel with low carbon content mostly used for heavy-duty machine parts, construction, and equipment, as well as the aviation and aerospace industry. It (and its substitutes) is characterized by its relatively high nickel content, and thus better tensile strength and yield point, as well as its very good hardenability, impact resistance, load deformation, wear, tension resistance, and high toughness. As for the specific test specimen, as seen in Figure 3, the central part, which is a tubular cylinder, allows us to obtain a quasi-homogeneous stress field and a negligible temperature gradient across its tube section.

Table 2. Chemical composition of the 35NCD16 steel used in the present study.

\begin{tabular}{cccccccccc}
\hline Element & $\mathbf{C}$ & $\mathbf{N i}$ & $\mathbf{C r}$ & $\mathbf{M o}$ & $\mathbf{M n}$ & $\mathbf{S i}$ & $\mathbf{P}$ & $\mathbf{S}$ & $\mathbf{F e}$ \\
\hline Weight [\%] & 0.34 & 3.67 & 1.54 & 0.31 & 0.35 & 0.26 & $<0.008$ & $<0.006$ & Balance \\
\hline
\end{tabular}




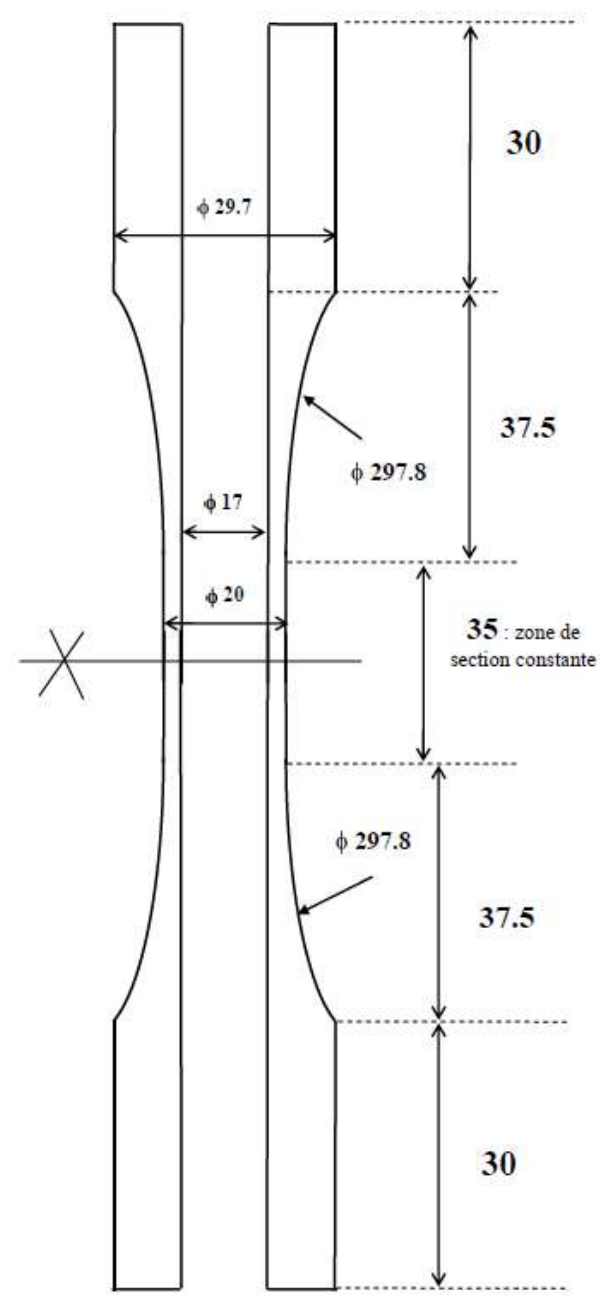

Figure 3. Dimensions (in mm) of 35NCD16 test specimen.

Before any test, the specimens undergo a heat treatment that leads to austenization in order to obtain the same initial mechanical and metallurgical state. This heat treatment consists of heating to $1050^{\circ} \mathrm{C}$, holding for one hour, followed by slow cooling in the furnace. The whole process is performed under vacuum.

\subsection{Elastoplastic Behavior of Austenite at Temperatures Close to Ms}

In order to conduct correct TRIP tests where the applied stress is lower than the yield stress of the austenitic phase in the temperature interval of the transformation, we need to have an idea about the yield limit at an adequate temperature. For this, we have performed a thermomechanical characterization at about $300{ }^{\circ} \mathrm{C}$ just before the onset of the martensitic transformation, namely, during cooling, when the temperature reaches $300^{\circ} \mathrm{C}$, a loading-unloading cycle is applied quickly (with a duration of about $2 \mathrm{~s}$ ). An example of the cycle carried out in the axial direction can be seen in Figure 4 where the yield stress of the austenitic phase may be estimated at about $160 \mathrm{MPa}$. Therefore, in order to be sure that the applied loading in our tests is in elasticity, we assume that the yield limit is around $120 \mathrm{MPa}$. The conditions of determination of this stress-strain diagram can be assumed to be isothermal regarding the small variation of temperature (less than $10^{\circ} \mathrm{C}$ ) during the application of the cycle (about $2 \mathrm{~s}$ ). 


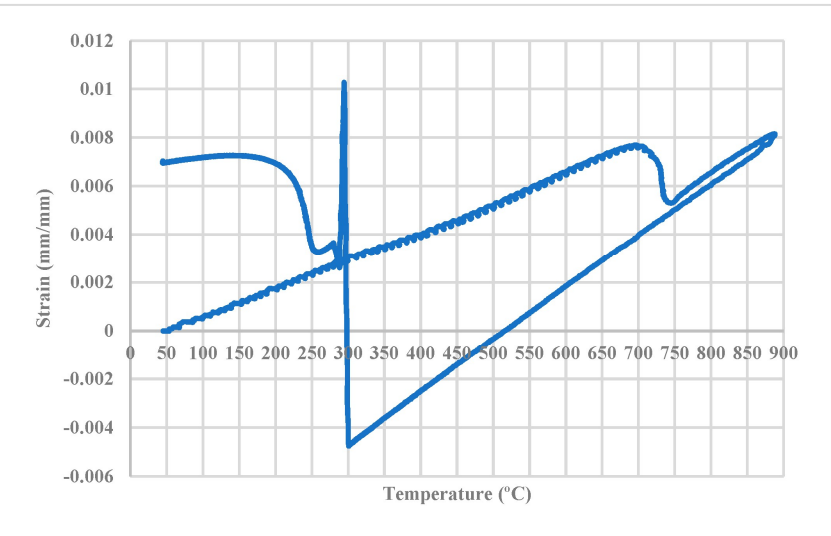

(a)

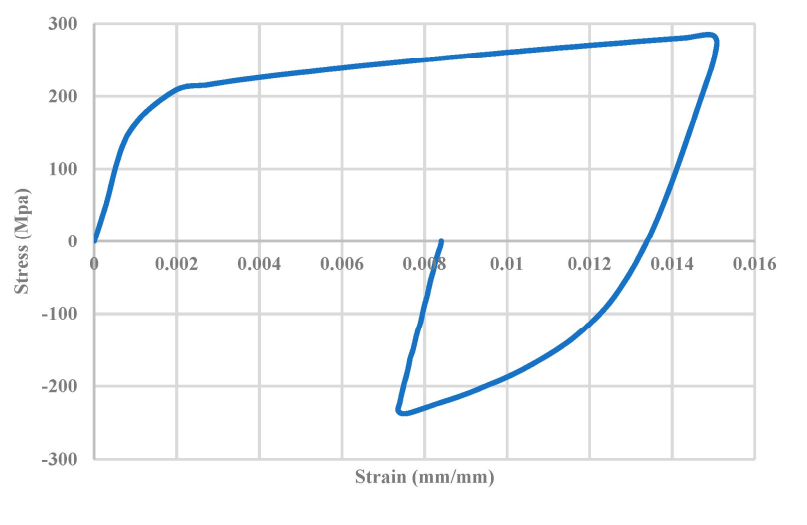

(b)

Figure 4. (a) Application of the loading-unloading cycle; (b) stress-strain diagram result of the loading-unloading cycle.

\section{Tests Performed and Obtained Results}

3.1. References: TRIP Tests Preceded Only by FD Tests

3.1.1. Method of Evaluation of TRIP

In a general assumption, evaluating TRIP consists of a relatively simple formula:

$$
\varepsilon^{t p}=\varepsilon^{t o t a l}-\varepsilon^{t h m}-\varepsilon^{e}
$$

where $\varepsilon^{t p}$ is the actual TRIP strain, $\varepsilon^{\text {total }}$ is the deformation measured during the TRIP test, $\varepsilon^{t h m}$ is the deformation measured during the FD test, and $\varepsilon^{e}$ is the measured elastic deformation. It is to be noted that this rule only applies when the stress applied to obtain TRIP is significantly smaller compared to the yield stress [4].

Knowing this, before evaluating TRIP, it is important to define the FD test, which will be considered as the reference. Indeed, FD test results obtained after applying TRIP tests (FD_2 in Figure 5) will not provide the same results as those that were performed before (FD_1). The graphs in Figure 5 for these two particular FD test cases show that after applying a TRIP test, there is a significant compressive plastic strain reducing the length of the specimen.

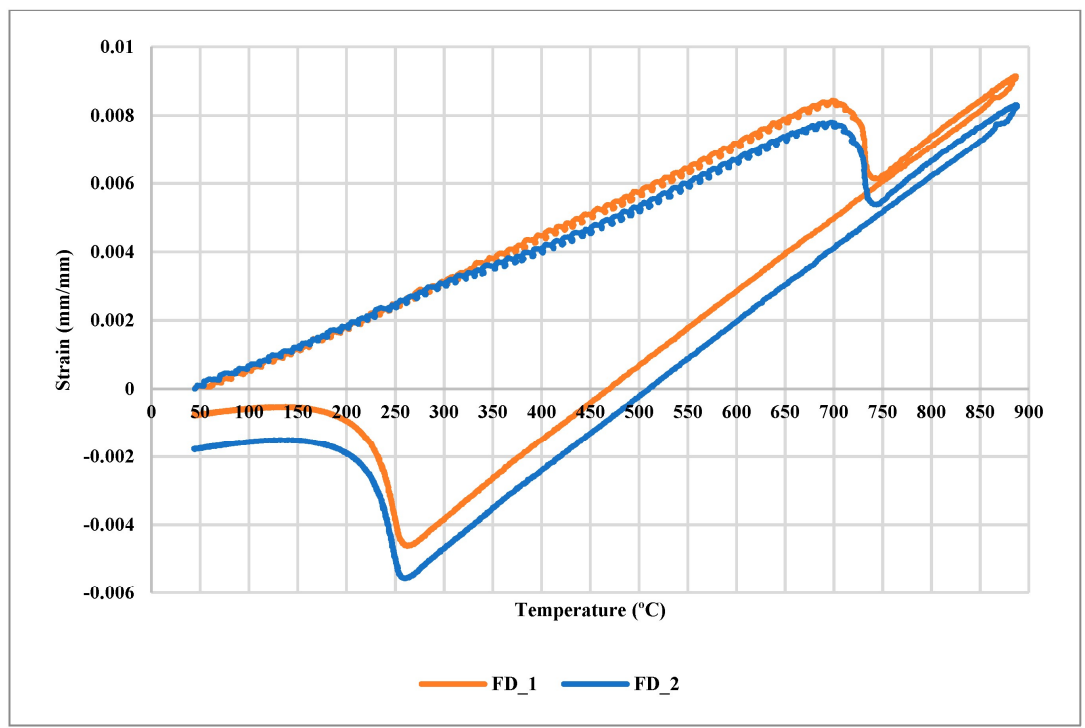

Figure 5. Free Dilatometric test results: FD_1 is carried out using virgin specimen while FD_2 is obtained using the same specimen subjected to TRIP cycle in addition to FD_1. 
However, taking a look at Figure 6, we see that, despite applying more TRIP tests, even for cases in which the stress applied to the specimen is higher, the following FD results (FD_3, FD_4, and FD_5) are almost superimposed. Therefore, FD_2, FD_3, FD_4, and FD_5 may be taken as references that may be used for the determination of TRIP.

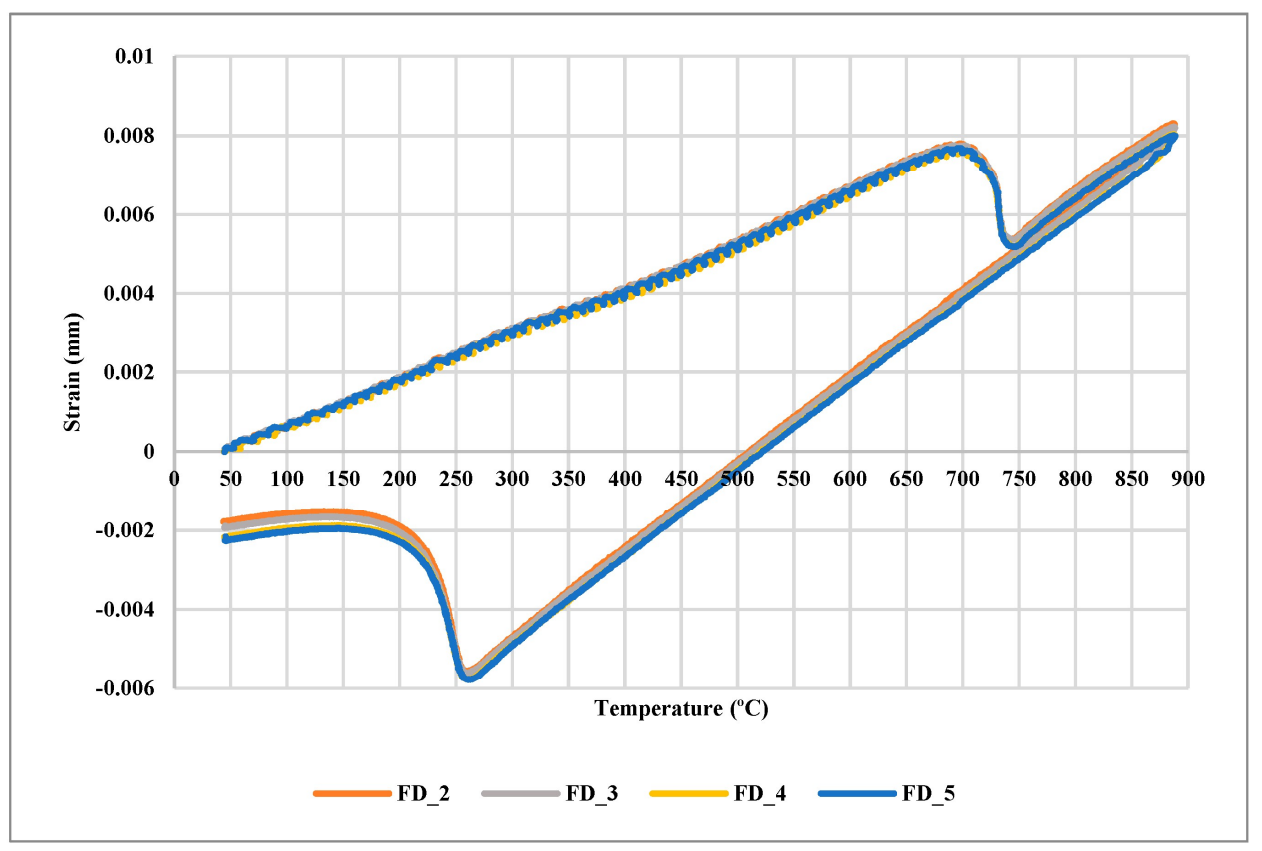

Figure 6. Free Dilatometric test results post TRIP tests.

This is where the importance of the choice of the Free Dilatometry results comes into play, as the results shown of the TRIP tests depend strongly on the results of the FD tests. Knowing this, we can proceed to evaluate our test specimens.

Three specimens were utilized, as was shown in the layout of tests to be performed. In the next section, we can see the TRIP test results for each test specimen only preceded by FD tests and they will be used as references for the following test results. Additionally, we present the comparison of the three reference TRIP test results to gain an idea of the magnitude of difference in the strain levels during tests.

\subsubsection{References of the TRIP Tests}

Here, we present the reference TRIP tests related to the levels of applied stresses. The reference of the TRIP test under $\sigma_{y} / 4 \approx 30 \mathrm{MPa}$ is denoted as $T_{\sigma_{y} / 4}^{r e f}$. Similarly, the reference of the TRIP tests performed with $\sigma_{y} / 2=60 \mathrm{MPa}$ and $3 \sigma_{y} / 4=90 \mathrm{MPa}$ are denoted as $T_{\sigma_{y} / 2}^{r e f}$ and $T_{3 \sigma_{y} / 4}^{r e f}$ respectively. We can see these aforementioned references in Figure 7. 


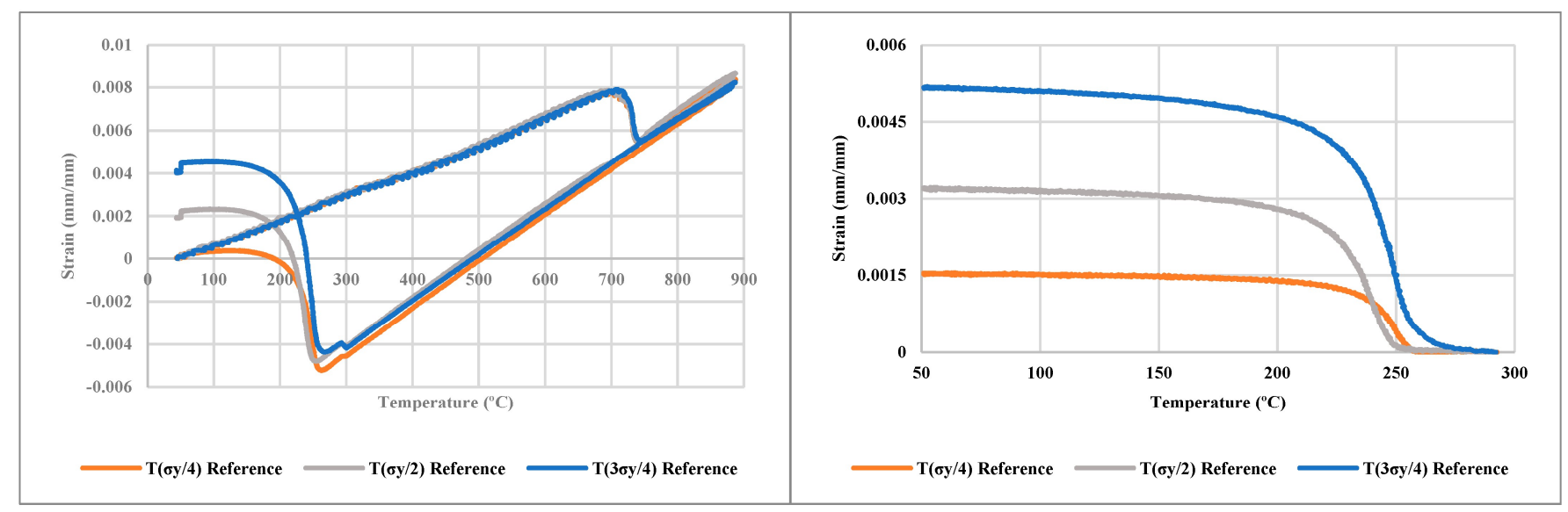

(a)

(b)

Figure 7. (a) Complete thermomechanical cycle comparison between $T_{\sigma_{y} / 4}^{r e f}, T_{\sigma_{y} / 2}^{r e f}$ and $T_{3 \sigma_{y} / 4} ;(\mathbf{b})$ TRIP strain vs. temperature comparison between $T_{\sigma_{y} / 4}^{r e f}, T_{\sigma_{y} / 2}^{r e f}$, and $T_{3 \sigma_{y} / 4}$. where:

To obtain these TRIP strain curves $\left(\varepsilon^{t p}\right)$ shown in Figure $7 b$, we refer back to Equation (1)

- $\quad \varepsilon^{\text {total }}$ : Deformation measured in the TRIP test.

- $\varepsilon^{t h m}$ : Deformation given by the FD test performed just before the TRIP test.

- $\quad \varepsilon^{e}:$ Elastic component, due to the applied stress, given by Hook's law.

As we are in a uniaxial stress state, $\varepsilon^{e}$ is given by Equation (2):

$$
\varepsilon^{e}=\frac{\sigma}{E}
$$

where:

- $\sigma$ : Applied mechanical (external) stress during the transformation.

- E: Young's modulus.

For instance, in the case of the test $T_{3 \sigma_{y} / 4}^{r e f}$ :

$\varepsilon^{\text {total }}$ is given in Figure 7a (blue) and $\varepsilon^{\text {thm }}$ is given in Figure 6, while $\varepsilon^{e}$ is equal to the ratio: $90 / 170,000=0.0005$.

Note that this method of determination of $\left(\varepsilon^{t p}\right)$ assumes that the applied loading does not significantly change the temperature Ms.

\subsection{Memory Effect for the Test-TRIP $\left(\sigma_{y} / 4\right)$}

To evaluate the memory effect, we compare our reference TRIP test characterized by the fact that the thermomechanical history applied on the specimen before the TRIP test is reduced in FD (see details in Section 3.1.2). Different cases were evaluated, which are detailed in the following subsections.

\subsubsection{Memory after Light Thermomechanical History Composed of One TRIP Test}

In this case, we compare the test results of $T_{\sigma_{y} / 4}^{r e f}$ with $T_{\sigma_{y} / 4}$. The details about thermomechanical history applied before $T_{\sigma_{y} / 4}$ are given in Table 3 . As for the test results, these can be seen in Figure 8. 
Table 3. Thermomechanical history applied before the test $T_{\sigma_{y} / 4}$ that will be compared to $T_{\sigma_{y} / 4}^{r e f}$.

\begin{tabular}{ccccc}
\hline $\mathbf{0}$ & $\mathbf{1}$ & $\mathbf{2}$ & $\mathbf{3}$ & $\mathbf{4}$ \\
\hline VS (Virgin specimen) & FD & $T_{\sigma_{y} / 4}^{r e f}$ & FD & $T_{\sigma_{y} / 4}$ \\
\hline
\end{tabular}

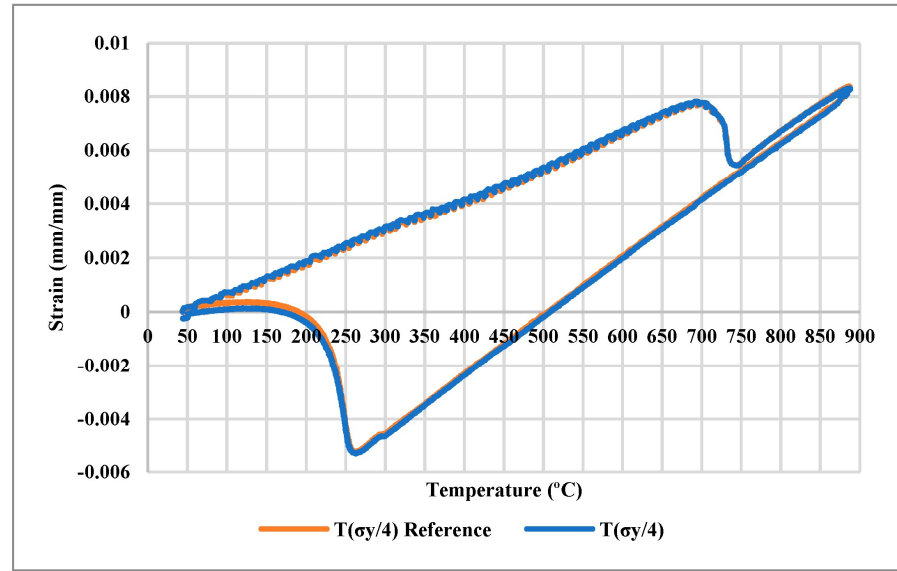

(a)

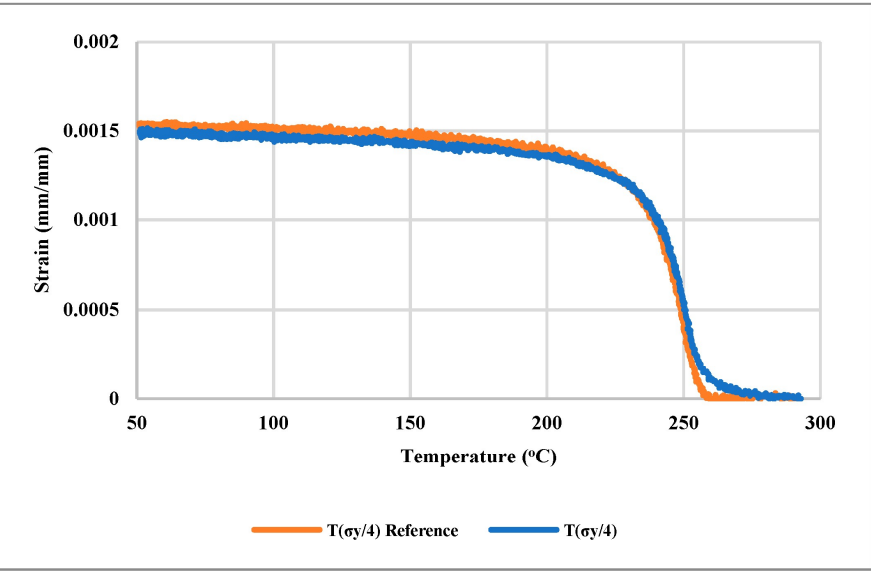

(b)

Figure 8. (a) Complete thermomechanical cycle comparison between $T_{\sigma_{y} / 4}^{r e f}$ and $T_{\sigma_{y} / 4}$ defined in Table 3; (b) TRIP strain vs. temperature comparison between $T_{\sigma_{y} / 4}^{r e f}$ and $T_{\sigma_{y} / 4}$.

The obtained results show that the two tests provide very similar behaviors; the two curves in Figure $8 \mathrm{~b}$ are almost perfectly superimposed.

\subsubsection{Memory after Medium Thermomechanical History Composed of 3 TRIP Tests}

Again, we compare the test results of $T_{\sigma_{y} / 4}^{r e f}$ with $T_{\sigma_{y} / 4}$. The details about thermomechanical history applied before $T_{\sigma_{y} / 4}$ are given in Table 4 . As for the test results, these can be seen in Figure 9.

Table 4. Thermomechanical history applied before the test $T_{\sigma_{y} / 4}$ that will be compared to $T_{\sigma_{y} / 4}^{r e f}$.

\begin{tabular}{ccccccccccc}
\hline $\mathbf{0}$ & $\mathbf{1}$ & $\mathbf{2}$ & $\mathbf{3}$ & $\mathbf{4}$ & $\mathbf{5}$ & $\mathbf{6}$ & $\mathbf{7}$ & $\mathbf{8}$ & $\mathbf{9}$ & $\mathbf{1 0}$ \\
\hline $\mathrm{VS}$ & $\mathrm{FD}$ & $T_{\sigma_{y} / 4}^{r e f}$ & $\mathrm{FD}$ & $T_{\sigma_{y} / 2}$ & FD & $T_{\sigma_{y} / 2}$ & FD & $T_{3 \sigma_{y} / 4}$ & FD & $T_{\sigma_{y} / 4}$ \\
\hline
\end{tabular}

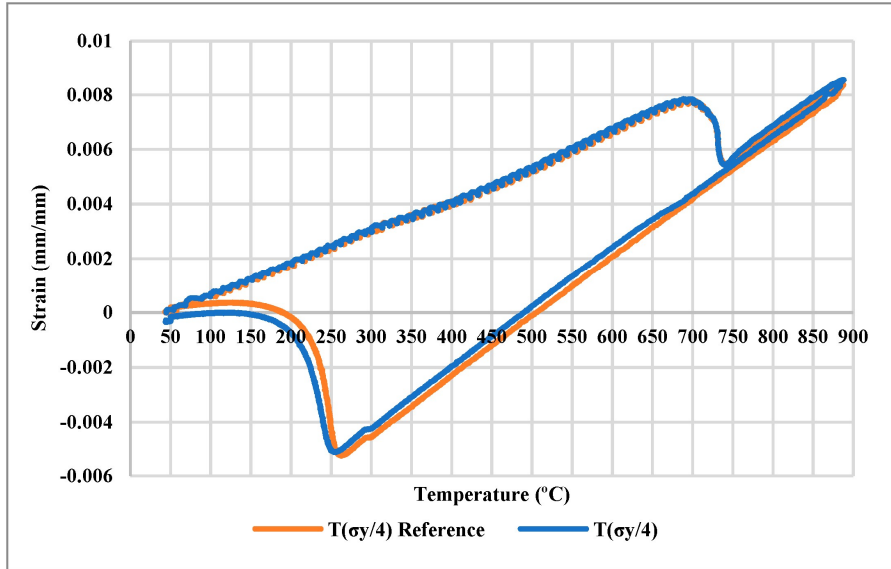

(a)

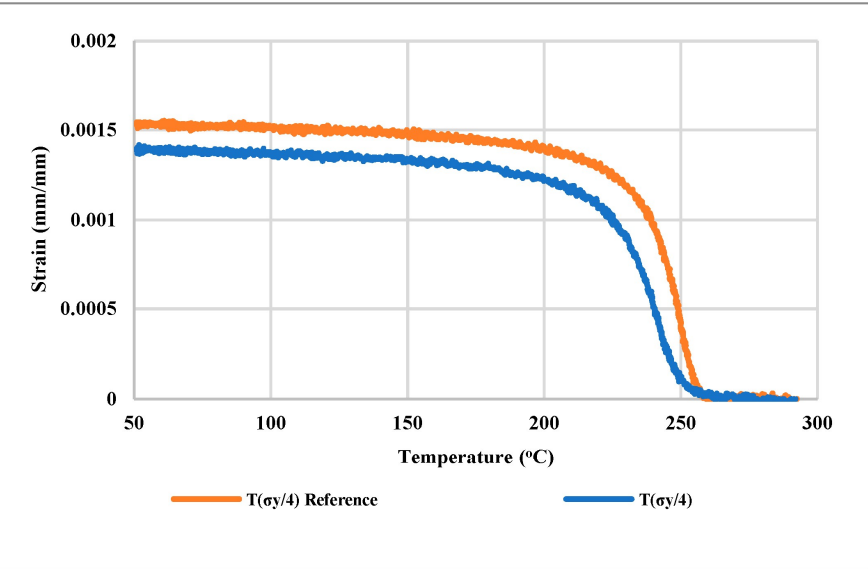

(b)

Figure 9. (a) Complete thermomechanical cycle comparison between $T_{\sigma_{y} / 4}^{r e f}$ and $T_{\sigma_{y} / 4}$ defined in Table 4 ; (b) TRIP strain vs. temperature comparison between $T_{\sigma_{y} / 4}^{r e f}$ and $T_{\sigma_{y} / 4}$. 
Unlike the previous case, the test $T_{\sigma_{y} / 4}$ leads to less TRIP than the reference test $T_{\sigma_{y} / 4}^{r e f}$.

\subsubsection{Memory after Medium Thermomechanical History Composed of 2 TRIP Tests}

In the present case, we compare the test result of $T_{\sigma_{y} / 4}^{r e f}$ with $T_{\sigma_{y} / 4}$. The details about thermomechanical history applied before $T_{\sigma_{y} / 4}$ are given in Table 5 . As for the test results, these can be seen in Figure 10.

Table 5. Thermomechanical history applied before the test $T_{\sigma_{y} / 4}$ that will be compared to $T_{\sigma_{y} / 4}^{r e f}$.

\begin{tabular}{ccccccccc}
\hline $\mathbf{0}$ & $\mathbf{1}$ & $\mathbf{2}$ & $\mathbf{3}$ & $\mathbf{4}$ & $\mathbf{5}$ & $\mathbf{6}$ & $\mathbf{7}$ & $\mathbf{8}$ \\
\hline $\mathrm{VS}$ & $\mathrm{FD}$ & $T_{\sigma_{y} / 4}^{r e f}$ & FD & $T_{3 \sigma_{y} / 4}$ & FD & $T_{3 \sigma_{y} / 4}$ & FD & $T_{\sigma_{y} / 4}$ \\
\hline
\end{tabular}

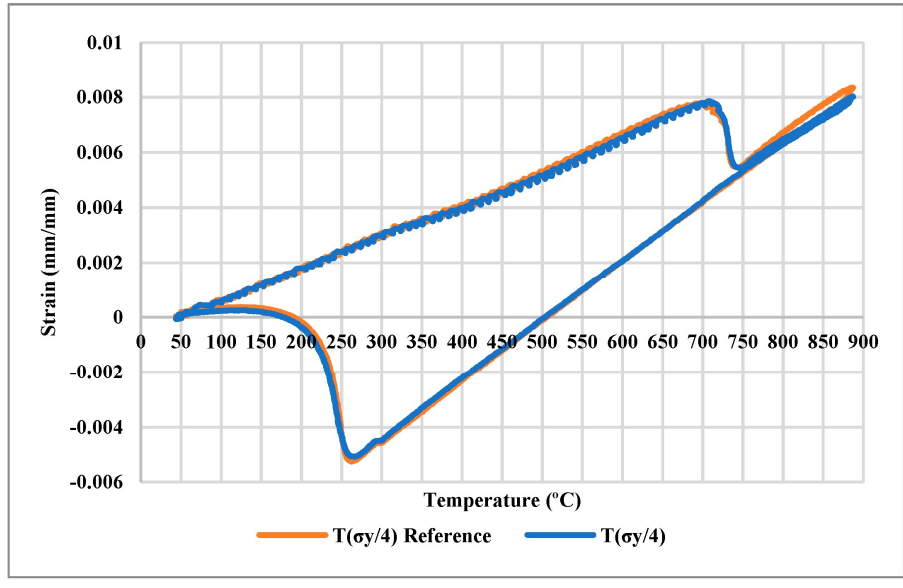

(a)

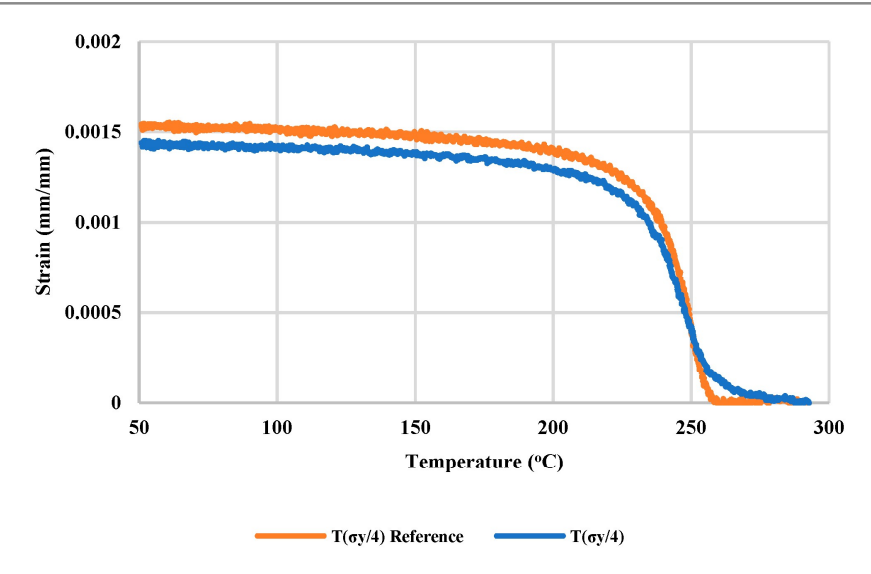

(b)

Figure 10. (a) Complete thermomechanical cycle comparison between $T_{\sigma_{y} / 4}^{r e f}$ and $T_{\sigma_{y} / 4}$ defined in Table 5; (b) TRIP strain v.stemperature comparison between $T_{\sigma_{y} / 4}^{r e f}$ and $T_{\sigma_{y} / 4}$.

The results here are very similar to the previous case.

3.2.4. Memory after Severe Thermomechanical History in Classical Elastoplasticity

In this section, we make some comparisons between $T_{\sigma_{y} / 4}^{r e f}$ and $T_{\sigma_{y} / 4}$ for cases in which $T_{\sigma_{y} / 4}$ is performed after the elastoplastic characterization cycle (Table 6). The comparison under question is given in Figure 11.

Table 6. Thermomechanical history applied before the test $T_{\sigma_{y} / 4}$ that will be compared to $T_{\sigma_{y} / 4}^{r e f}$.

\begin{tabular}{ccccccc}
\hline $\mathbf{0}$ & $\mathbf{1}$ & $\mathbf{2}$ & $\mathbf{3}$ & $\mathbf{4}$ & $\mathbf{5}$ & $\mathbf{6}$ \\
\hline VS & FD & $T_{\sigma_{y} / 4}^{r e f}$ & FD & Elastoplastic cycle (see Figure 4) & FD & $T_{\sigma_{y} / 4}$ \\
\hline
\end{tabular}




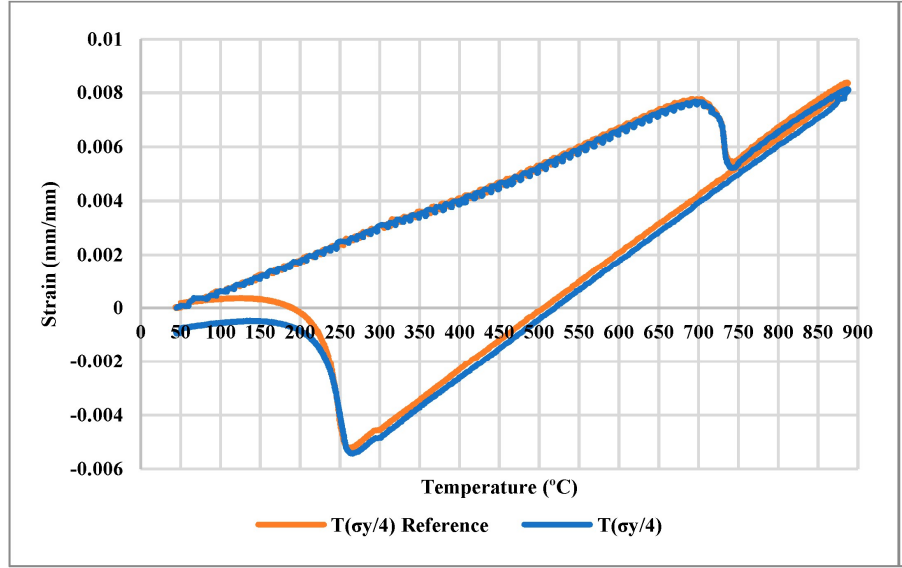

(a)

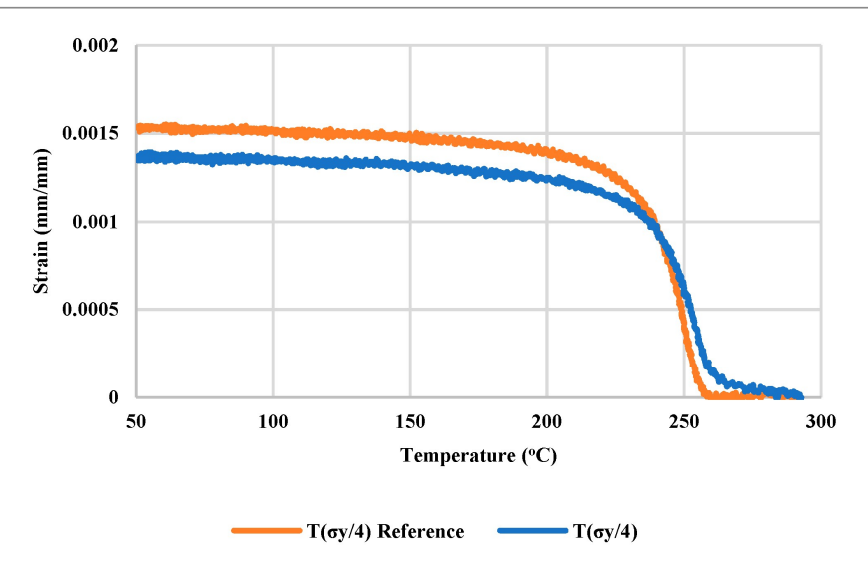

(b)

Figure 11. (a) Complete thermomechanical cycle comparison between $T_{\sigma_{y} / 4}^{r e f}$ and $T_{\sigma_{y} / 4}$ defined in Table 6; (b) TRIP strain vs. temperature comparison between $T_{\sigma_{y} / 4}^{r e f}$ and $T_{\sigma_{y} / 4}$.

Once again, the results here are very similar to the previous case.

\subsection{Memory Effect for the Test-TRIP $\left(\sigma_{y} / 2\right)$}

3.3.1. Memory after Light Thermomechanical History Composed of One TRIP Test

In this case, we compare the test results of $T_{\sigma_{y} / 2}^{r e f}$ with $T_{\sigma_{y} / 2}$. The details about thermomechanical history applied before $T_{\sigma_{y} / 4}$ are given in Table 7 . As for the test results, these can be seen in Figure 12.

Table 7. Thermomechanical history applied before the test $T_{\sigma_{y} / 2}$ that will be compared to $T_{\sigma_{y} / 2}^{r e f}$.

\begin{tabular}{ccccc}
\hline $\mathbf{0}$ & $\mathbf{1}$ & $\mathbf{2}$ & $\mathbf{3}$ & $\mathbf{4}$ \\
\hline $\mathrm{VS}$ & FD & $T_{\sigma_{y} / 2}^{r e f}$ & FD & $T_{\sigma_{y} / 2}$ \\
\hline
\end{tabular}

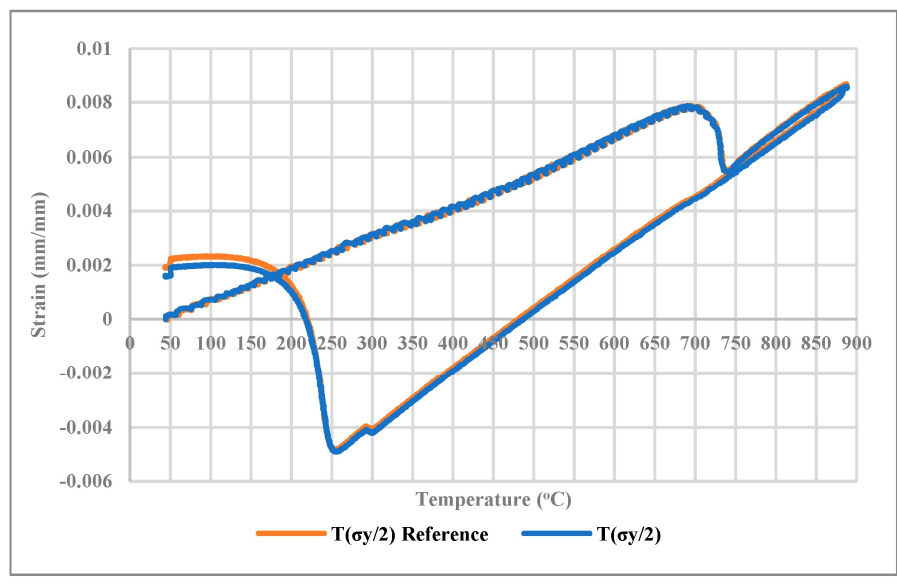

(a)

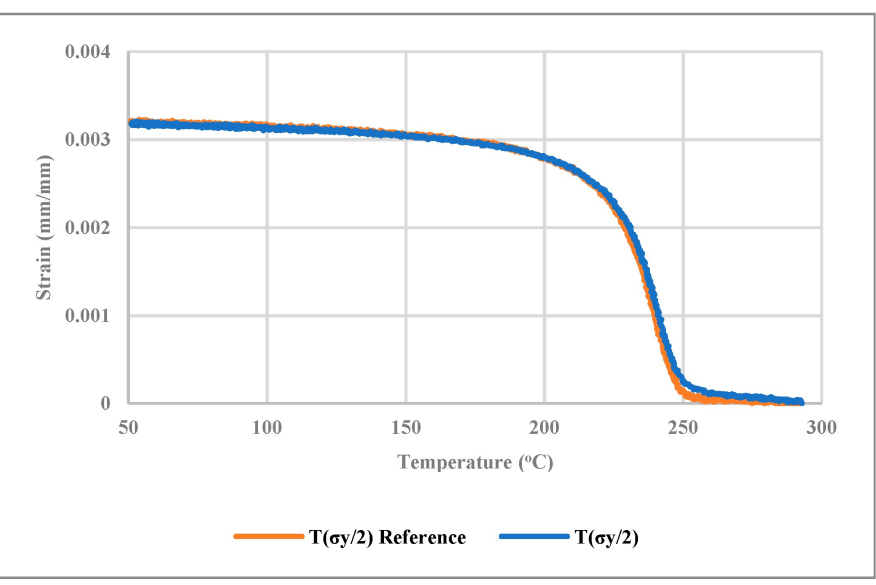

(b)

Figure 12. (a) Complete thermomechanical cycle comparison between $T_{\sigma_{y} / 2}^{r e f}$ and $T_{\sigma_{y} / 2}$ defined in Table 7; (b) TRIP strain vs. temperature comparison between $T_{\sigma_{y} / 2}^{r e f}$ and $T_{\sigma_{y} / 2}$. 
The obtained result is very similar to the corresponding one using $\sigma_{y} / 4$ described in Section 3.2.1 (Figure 8). No effect at all of the applied thermomechanical history occurred on the TRIP.

\subsubsection{Memory after Medium Thermomechanical History Composed of 3 TRIP Tests}

Again, we compare the test results of $T_{\sigma_{y} / 2}^{r e f}$ with $T_{\sigma_{y} / 2}$, but in this case, there is more thermomechanical history applied to the test specimen between the two results to compare. The details about thermomechanical history applied before $T_{\sigma_{y} / 2}$ are given in Table 8 . As for the test results, these can be seen in Figure 13.

Table 8. Thermomechanical history applied before the test $T_{\sigma_{y} / 2}$ that will be compared to $T_{\sigma_{y} / 2}^{r e f}$.

\begin{tabular}{ccccccccccc}
\hline $\mathbf{0}$ & $\mathbf{1}$ & $\mathbf{2}$ & $\mathbf{3}$ & $\mathbf{4}$ & $\mathbf{5}$ & $\mathbf{6}$ & $\mathbf{7}$ & $\mathbf{8}$ & $\mathbf{9}$ & $\mathbf{1 0}$ \\
\hline $\mathrm{VS}$ & $\mathrm{FD}$ & $T_{\sigma_{y} / 2}^{r e f}$ & $\mathrm{FD}$ & $T_{3 \sigma_{y} / 4}$ & FD & $T_{3 \sigma_{y} / 4}$ & FD & $T_{\sigma_{y} / 4}$ & FD & $T_{\sigma_{y} / 2}$ \\
\hline
\end{tabular}

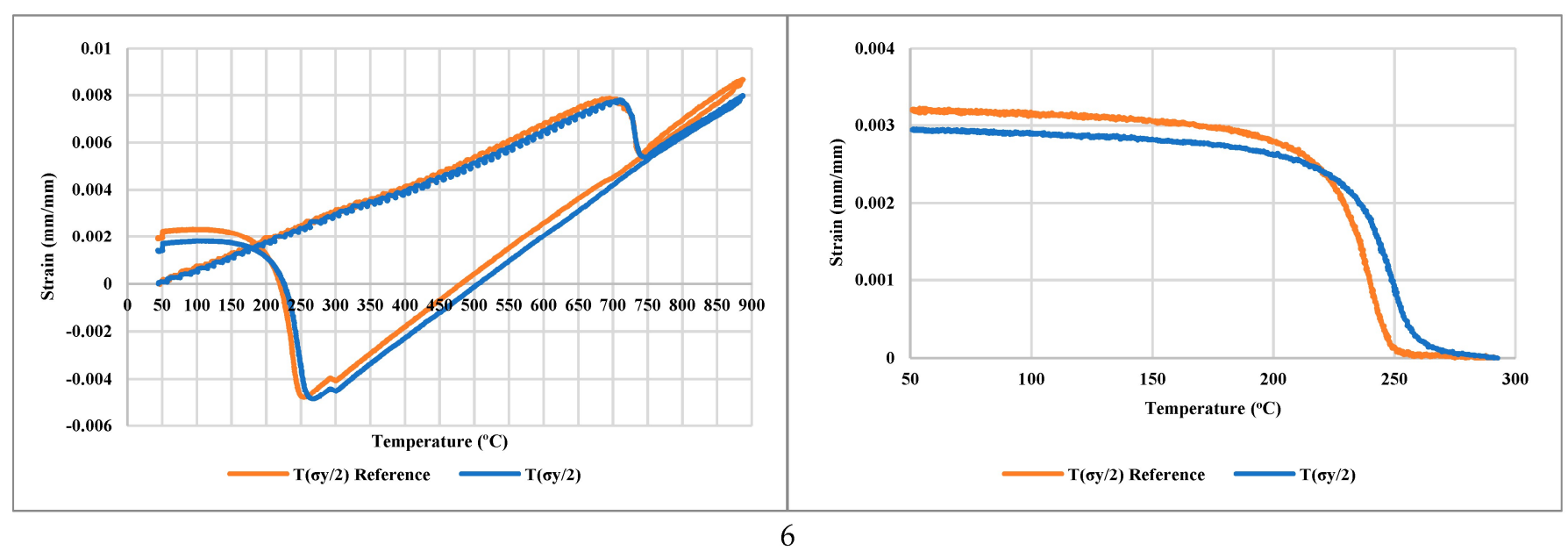

(a)

(b)

Figure 13. (a) Complete thermomechanical cycle comparison between $T_{\sigma_{y} / 2}^{r e f}$ and $T_{\sigma_{y} / 2}$ defined in Table 8; (b) TRIP strain vs. temperature comparison between $T_{\sigma_{y} / 2}^{r e f}$ and $T_{\sigma_{y} / 2}$.

In this case, the martensitic transformation starts a little bit before compared to $T_{\sigma_{y} / 2}^{r e f}$

\subsubsection{Memory after Medium Thermomechanical History Composed of 2 TRIP Tests}

Our third comparison of the test results of $T_{\sigma_{y} / 2}^{r e f}$ with $T_{\sigma_{y} / 2}$ also shows a high amount of thermomechanical history applied to the test specimen compared to the case of Section 3.3.1 between the two results to compare. The details about thermomechanical history applied before $T_{\sigma_{y} / 2}$ are given in Table 9 . As for the test results, these can be seen in Figure 14 .

Table 9. Thermomechanical history applied before the test $T_{\sigma_{y} / 2}$ that will be compared to $T_{\sigma_{y} / 2}^{r e f}$.

\begin{tabular}{ccccccccc}
\hline $\mathbf{0}$ & $\mathbf{1}$ & $\mathbf{2}$ & $\mathbf{3}$ & $\mathbf{4}$ & $\mathbf{5}$ & $\mathbf{6}$ & $\mathbf{7}$ & $\mathbf{8}$ \\
\hline $\mathrm{VS}$ & $\mathrm{FD}$ & $T_{\sigma_{y} / 2}^{r e f}$ & FD & $T_{\sigma_{y} / 4}$ & FD & $T_{\sigma_{y} / 4}$ & FD & $T_{\sigma_{y} / 2}$ \\
\hline
\end{tabular}




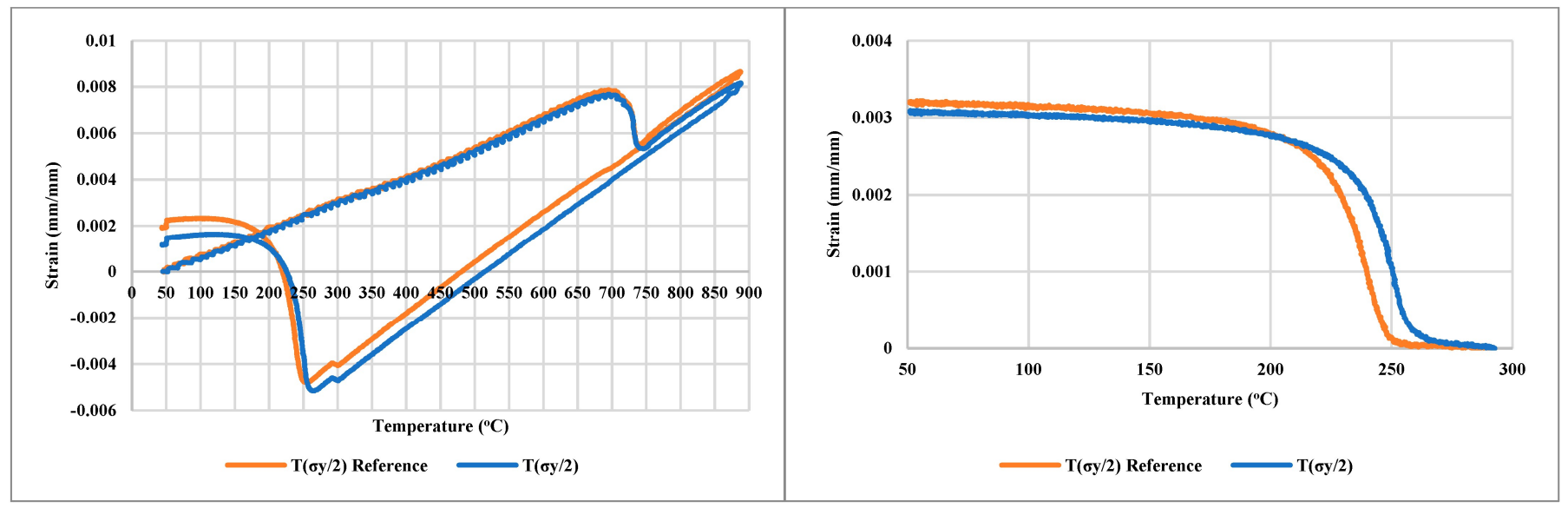

(a)

(b)

Figure 14. (a) Complete thermomechanical cycle comparison between $T_{\sigma_{y} / 2}^{r e f}$ and $T_{\sigma_{y} / 2}$ defined in Table 9; (b) TRIP strain vs. temperature comparison between $T_{\sigma_{y} / 2}^{r e f}$ and $T_{\sigma_{y} / 2}$.

Once again, the martensitic transformation starts a little bit before the case of the reference.

\subsubsection{Memory after Severe Thermomechanical History in Classical Elastoplasticity}

In this section, we make some comparisons between $T_{\sigma_{y} / 2}^{r e f}$ and $T_{\sigma_{y} / 2}$ for cases in which $T_{\sigma_{y} / 2}$ is performed after the elastoplastic characterization cycle (Table 10). The comparison under question is given in Figure 15.

Table 10. Thermomechanical history applied before the test $T_{\sigma_{y} / 2}$ that will be compared to $T_{\sigma_{y} / 2}^{r e f}$.

\begin{tabular}{ccccccc}
\hline $\mathbf{0}$ & $\mathbf{1}$ & $\mathbf{2}$ & $\mathbf{3}$ & $\mathbf{4}$ & $\mathbf{5}$ & $\mathbf{6}$ \\
\hline VS & FD & $T_{\sigma_{y} / 2}^{r e f}$ & FD & Elastoplastic cycle (see Figure 4) & FD & $T_{\sigma_{y} / 2}$ \\
\hline
\end{tabular}

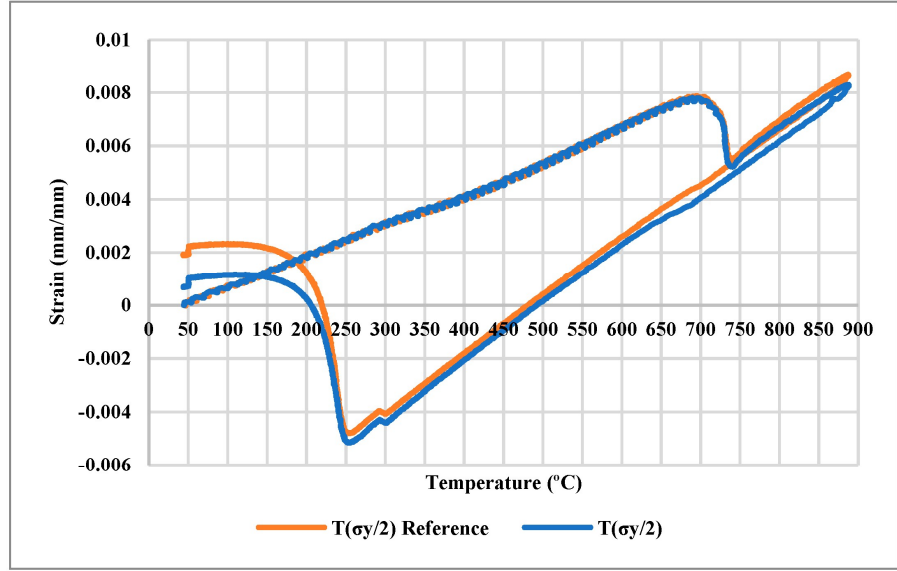

(a)

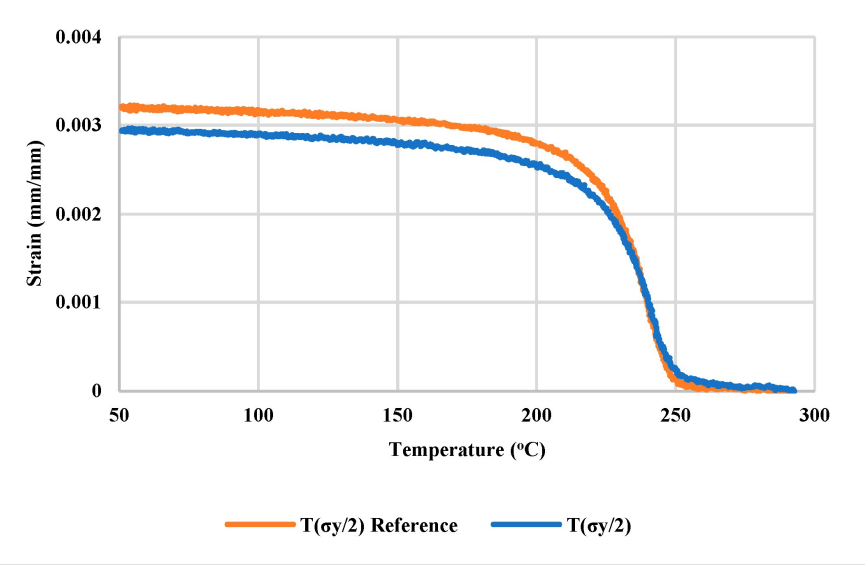

(b)

Figure 15. (a) Complete thermomechanical cycle comparison between $T_{\sigma_{y} / 2}^{r e f}$ and $T_{\sigma_{y} / 2}$ defined in Table 10; (b) TRIP strain vs. temperature comparison between $T_{\sigma_{y} / 2}^{r e f}$ and $T_{\sigma_{y} / 2}$.

The results show very small difference between the amount of TRIP in the two tests. 


\subsection{Memory Effect for the Test-TRIP $\left(3 \sigma_{y} / 4\right)$}

\subsubsection{Memory after Light Thermomechanical History Composed of One TRIP Test}

In this case, we compare the test results of $T_{3 \sigma_{y} / 4}^{r e f}$ with $T_{3 \sigma_{y} / 4}$. The details about thermomechanical history applied before $T_{3 \sigma_{y} / 4}$ are given in Table 11 . As for the test results, these can be seen in Figure 16.

Table 11. Thermomechanical history applied before the test $T_{3 \sigma_{y} / 4}$ that will be compared to $T_{3 \sigma_{y} / 4}^{r e f}$.

\begin{tabular}{ccccc}
\hline $\mathbf{0}$ & $\mathbf{1}$ & $\mathbf{2}$ & $\mathbf{3}$ & $\mathbf{4}$ \\
\hline VS & FD & $T_{3 \sigma_{y} / 4}^{r e f}$ & FD & $T_{3 \sigma_{y} / 4}$ \\
\hline
\end{tabular}

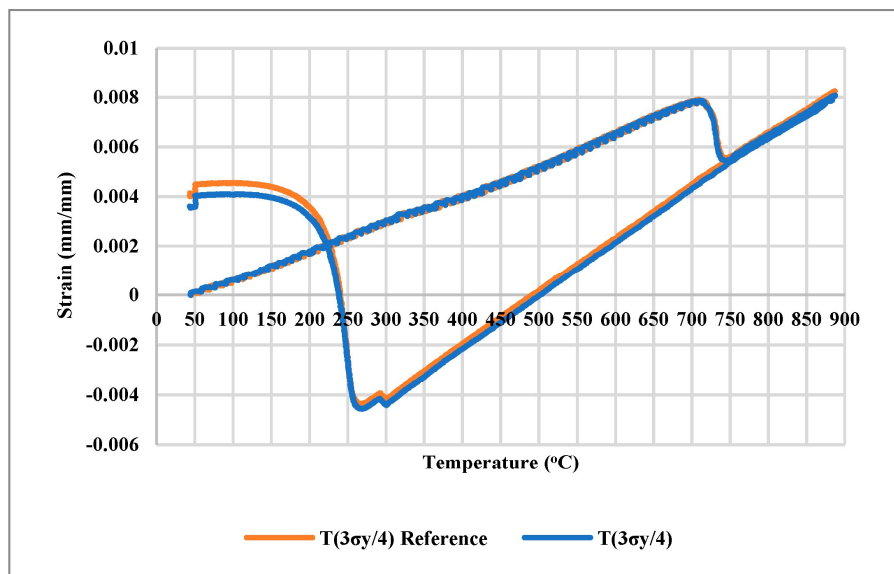

(a)

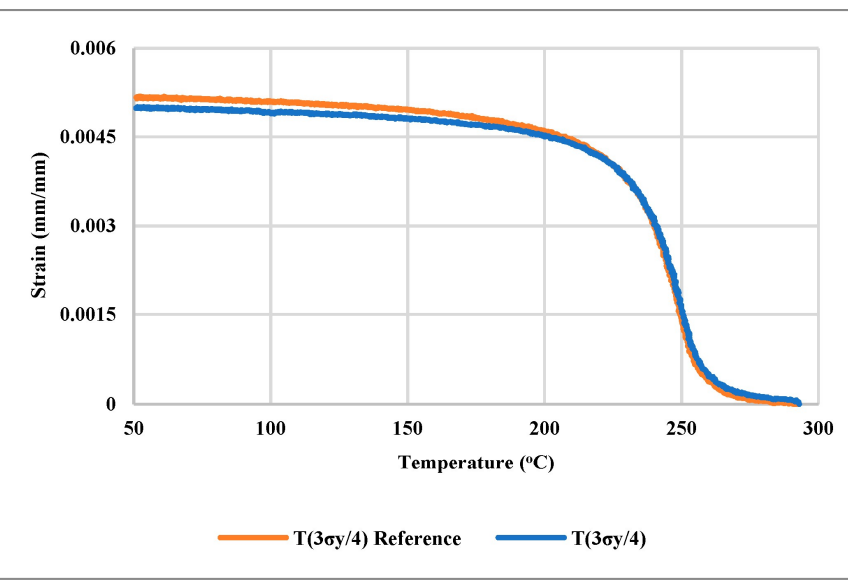

(b)

Figure 16. (a) Complete thermomechanical cycle comparison between $T_{3 \sigma_{y} / 4}^{r e f}$ and $T_{3 \sigma_{y} / 4}$ defined in Table 11; (b) TRIP strain vs. temperature comparison between $T_{3 \sigma_{y} / 4}^{r e f}$ and $T_{3 \sigma_{y} / 4}$.

The obtained results show that the two tests provide very similar behaviors; the two curves in Figure $16 \mathrm{~b}$ are almost perfectly superimposed. This result is very similar to the equivalent ones with $\sigma_{y} / 4$ (Figure 8 ) and $\sigma_{y} / 2$ (Figure 12).

\subsubsection{Memory after Medium Thermomechanical History Composed of 3 TRIP Tests}

Again, we compare the test results of $T_{3 \sigma_{y} / 4}^{r e f}$ with $T_{3 \sigma_{y} / 4}$, but in this case, there is more thermomechanical history applied to the test specimen between the two results to compare. The details about thermomechanical history applied before $T_{3 \sigma_{y} / 4}$ are given in Table 12. As for the test results, these can be seen in Figure 17.

Table 12. Thermomechanical history applied before the test $T_{3 \sigma_{y} / 4}$ that will be compared to $T_{3 \sigma_{y} / 4}^{r e f}$.

\begin{tabular}{ccccccccccc}
\hline $\mathbf{0}$ & $\mathbf{1}$ & $\mathbf{2}$ & $\mathbf{3}$ & $\mathbf{4}$ & $\mathbf{5}$ & $\mathbf{6}$ & $\mathbf{7}$ & $\mathbf{8}$ & $\mathbf{9}$ & $\mathbf{1 0}$ \\
\hline VS & FD & $T_{3 \sigma_{y} / 4}^{r e f}$ & FD & $T_{\sigma_{y} / 4}$ & FD & $T_{\sigma_{y} / 4}$ & FD & $T_{\sigma_{y} / 2}$ & FD & $T_{3 \sigma_{y} / 4}$ \\
\hline
\end{tabular}




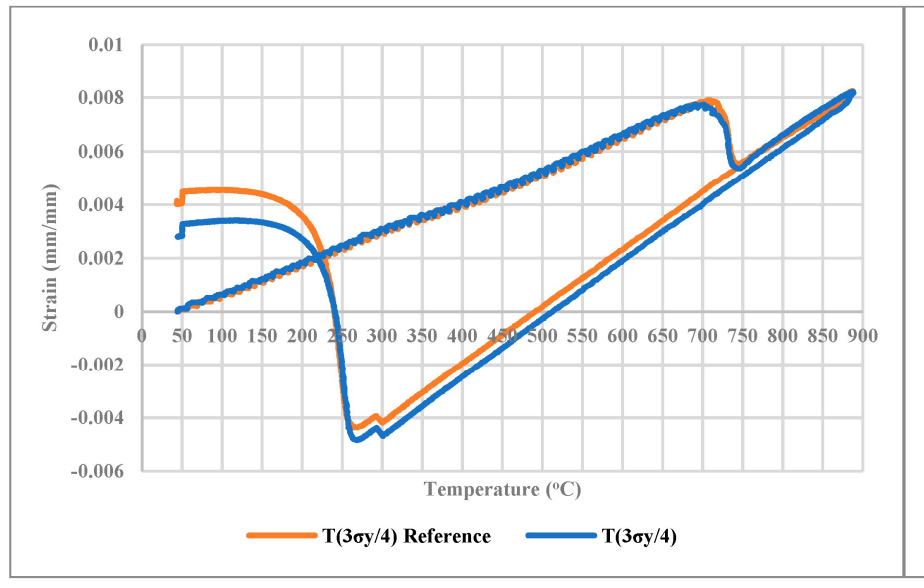

(a)

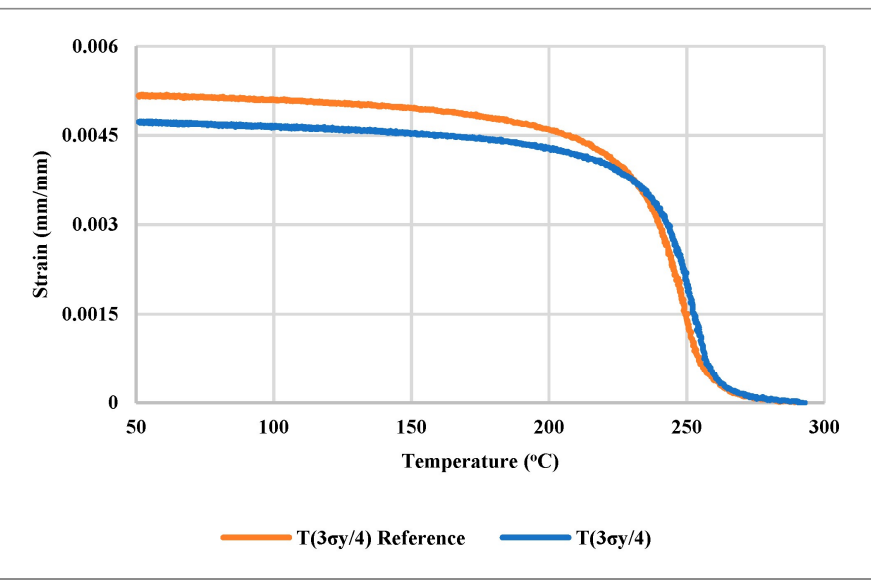

(b)

Figure 17. (a) Complete thermomechanical cycle comparison between $T_{3 \sigma_{y} / 4}^{r e f}$ and $T_{3 \sigma_{y} / 4}$ defined in Table 12; (b) TRIP strain vs. temperature comparison between $T_{3 \sigma_{y} / 4}^{r e f}$ and $T_{3 \sigma_{y} / 4}$.

Similar to the previous section, the TRIP evolves with the same manner in both cases, no significant effect of the applied thermomechanical history is observed.

\subsubsection{Memory after Medium Thermomechanical History Composed of 2 TRIP Tests}

Our third comparison of the test results of $T_{3 \sigma_{y} / 4}^{r e f}$ with $T_{3 \sigma_{y} / 4}$ also shows a high amount of thermomechanical history applied to the test specimen compared to the case of Section 3.4.1 between the two results to compare. The details about thermomechanical history applied before $T_{3 \sigma_{y} / 4}$ are given in Table 13. As for the test results, these can be seen in Figure 18.

Table 13. Thermomechanical history applied before the test $T_{3 \sigma_{y} / 4}$ that will be compared to $T_{3 \sigma_{y} / 4}^{r e f}$.

\begin{tabular}{ccccccccc}
\hline $\mathbf{0}$ & $\mathbf{1}$ & $\mathbf{2}$ & $\mathbf{3}$ & $\mathbf{4}$ & $\mathbf{5}$ & $\mathbf{6}$ & $\mathbf{7}$ & $\mathbf{8}$ \\
\hline VS & FD & $T_{3 \sigma_{y} / 4}^{r e f}$ & FD & $T_{\sigma_{y} / 2}$ & FD & $T_{\sigma_{y} / 2}$ & FD & $T_{3 \sigma_{y} / 4}$ \\
\hline
\end{tabular}

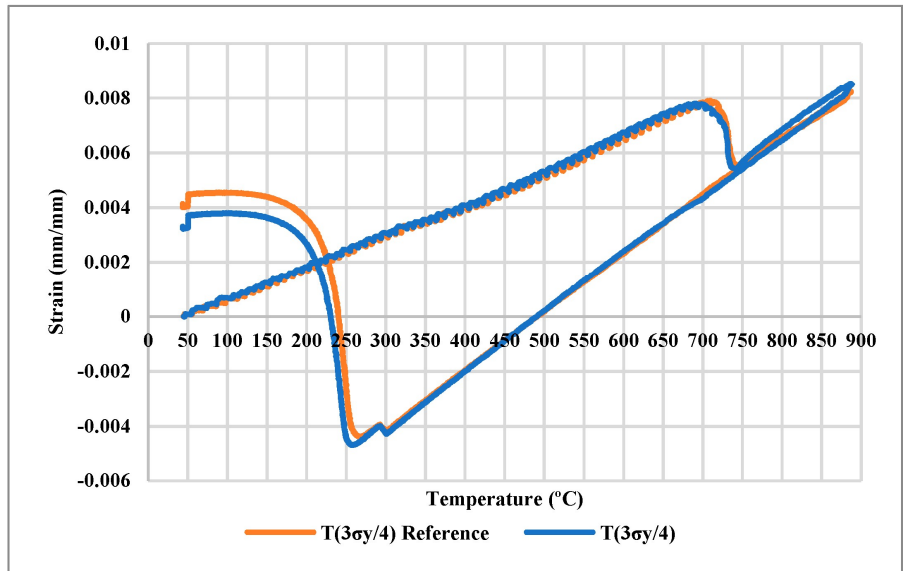

(a)

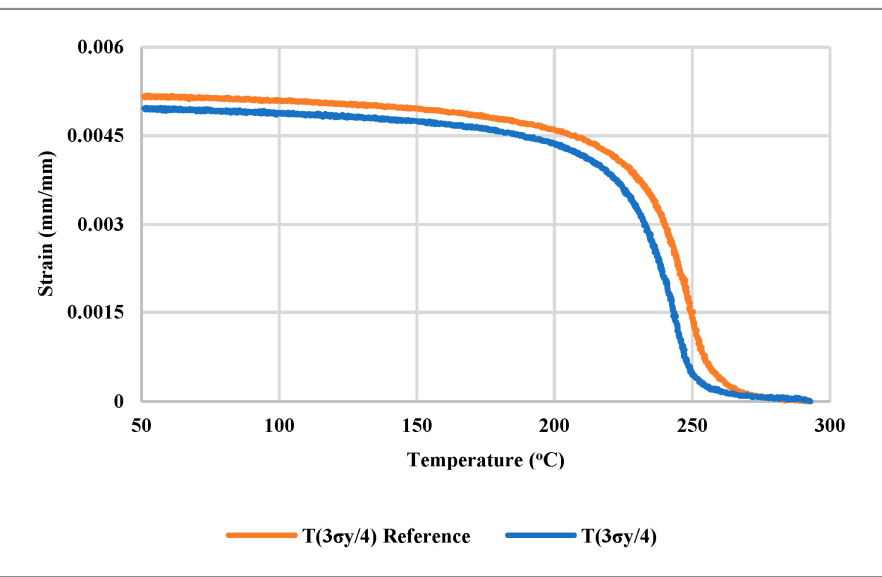

(b)

Figure 18. (a) Complete thermomechanical cycle comparison between $T_{3 \sigma_{y} / 4}^{r e f}$ and $T_{3 \sigma_{y} / 4}$ defined in Table 13; (b) TRIP strain vs. temperature comparison between $T_{3 \sigma_{y} / 4}^{r e f}$ and $T_{3 \sigma_{y} / 4}$. 
Similar results were obtained for both tests, despite the fact that in the present, the martensitic transformation seems to start earlier.

\subsubsection{Memory after Severe Thermomechanical History in Classical Elastoplasticity}

In this section, we make some comparisons between $T_{3 \sigma_{y} / 4}^{r e f}$ and $T_{3 \sigma_{y} / 4}$ for cases in which $T_{3 \sigma_{y} / 4}$ is performed after the elastoplastic characterization cycles (Table 14). The comparison under question is given in Figure 19.

Table 14. Thermomechanical history applied before the test $T_{3 \sigma_{y} / 4}$ that will be compared to $T_{3 \sigma_{y} / 4}^{r e f}$.

\begin{tabular}{ccccccc}
\hline $\mathbf{0}$ & $\mathbf{1}$ & $\mathbf{2}$ & $\mathbf{3}$ & $\mathbf{4}$ & $\mathbf{5}$ & $\mathbf{6}$ \\
\hline VS & FD & $T_{3 \sigma_{y} / 4}^{r e f}$ & FD & Elastoplastic cycle (see Figure 4) & FD & $T_{3 \sigma_{y} / 4}$ \\
\hline
\end{tabular}

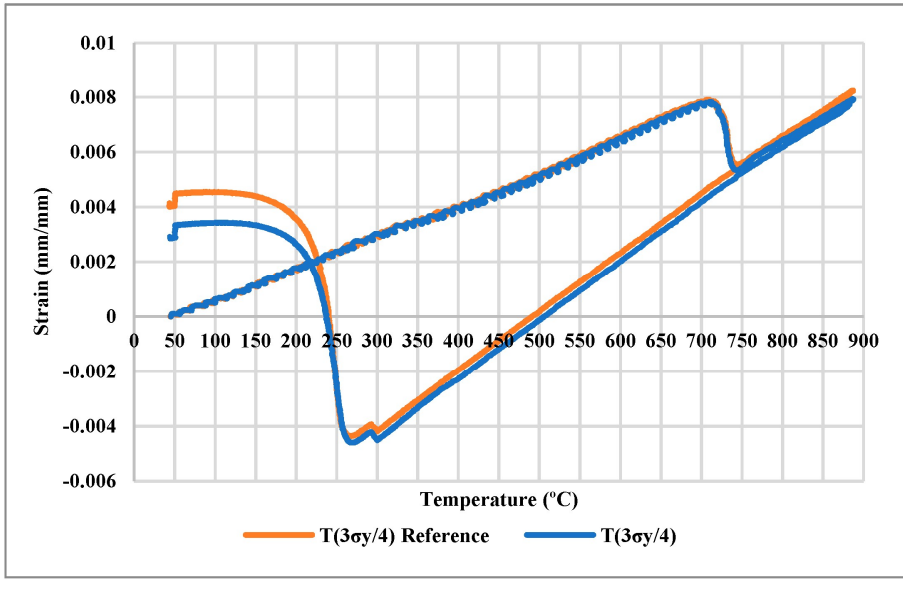

(a)

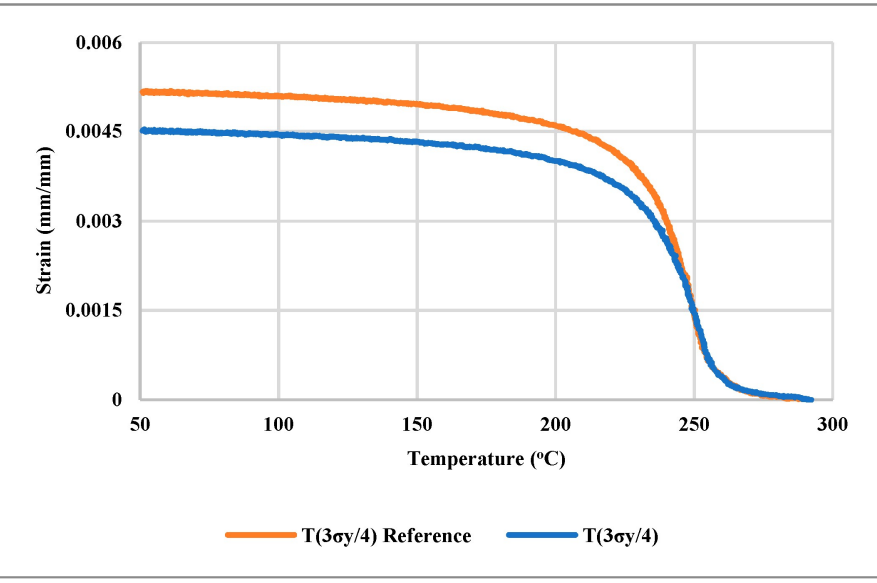

(b)

Figure 19. (a) Complete thermomechanical cycle comparison between $T_{3 \sigma_{y} / 4}^{r e f}$ and $T_{3 \sigma_{y} / 4}$ defined in Table 14; (b) TRIP strain vs. temperature comparison between $T_{3 \sigma_{y} / 4}^{r e f}$ and $T_{3 \sigma_{y} / 4}$.

Similar to the cases of Sections 3.2.4 and 3.3.4, the results show us that the case after the characterization cycle leads to less TRIP.

\subsection{Analysis of Other TRIP Characteristics}

\subsubsection{Effect on the Kinetics of Transformation Plasticity}

The kinetics of TRIP are usually expressed by the function defined by the ratio vs. the volume proportion $(\mathrm{Z})$ of the martensite phase that is formed during these tests:

$$
\left(\varepsilon^{t p}(Z) /\left(\varepsilon^{t p}(Z=1)\right)\right.
$$

This function is estimated from experimental results,

$$
\varepsilon^{t p}=f(T)
$$

and the volume proportion of the new formed phase $(\alpha)$ vs. temperature

$$
Z=f(T) .
$$


Equation (5) is estimated from thermal strain evolution vs. temperature (Equation (4) deducted from free dilatometry test) by assuming the linear mixture rule to obtain the thermal strain $\left(\varepsilon^{t h m}\right)$ of the phases mixture [4]. The thermal strain can be expressed by

$$
\varepsilon^{\text {thm }}=\alpha \cdot\left(T-T^{\alpha}\right)+(1-Z) \cdot \varepsilon_{M A}^{T^{\alpha}}
$$

with

$$
\alpha=Z \cdot \alpha_{M}+(1-Z) \cdot \alpha_{A}
$$

where $Z$ is the volume fraction of the martensitic phase, $\alpha_{M}$ is the dilatation coefficient of the martensitic phase, $\alpha_{A}$ is the dilatation coefficient of the austenitic phase, $\alpha$ is the dilatation coefficient of the mixture, $\Delta \varepsilon_{M A}^{T^{\alpha}}$ is the compactness variation (also known as deformation of transformation) between the martensitic and the austenitic phases at room temperature, which, for our case, varies between $0.63 \%$ and $0.88 \%$ at room temperature at the beginning of the transformation, and $T^{\alpha}$ is the references temperature of $\alpha$ phases.

Equation (6) and Equation (7) lead to

$$
Z=\frac{\varepsilon^{t h m}-\alpha_{A} \cdot\left(T-T^{\alpha}\right)-\Delta \varepsilon_{M A}^{T^{\alpha}}}{\left(\alpha_{M}-\alpha_{A}\right) \cdot\left(T-T^{\alpha}\right)-\Delta \varepsilon_{M A}^{T^{\alpha}}}
$$

With this expression, we can now estimate Equation (3), which will provide us the transformation plasticity evolution vs. the martensitic volume proportion for specific tests.

\subsubsection{TRIP Parameter $k$}

In the cases where the applied stress is small, uniaxial, and maintained constant during the transformation, certain TRIP models, such as Desalos' model for example, may be expressed by the following equation:

$$
\varepsilon^{t p}=k z(2-z) \sigma
$$

where $k$ is generally known as the TRIP parameter. This equation is valid if the applied load remains constant during the transformation, for which a rate formulation is necessary in general.

This parameter can be defined as a function of the transformation progress [2]. It allows us to analyze the TRIP rate of a test specimen as a function of the volume fraction of the product phase and the applied stress to it [1]. This parameter can be estimated experimentally using tests where applied stress does not generate classical plasticity or creep, which is why in most cases this is determined using test results that do not surpass half of the test specimen's yield stress.

Equation (9) leads to

$$
k=\frac{\varepsilon^{t p}}{z(2-z) \sigma} ; \text { if } z=1, k=\frac{\varepsilon_{f}^{t p}}{\sigma}
$$

In these conditions, $k$ is constant. Because of this, it may be determined after a TRIP test as the ratio between the final TRIP and the applied stress.

Desalos' model [10] uses such a method in order to determine the coefficient $k$. This character makes this model non-predictive.

One can find in the literature the expression giving the parameter $k$ based on micromechanical considerations, such as in Leblond (1989) [15], who presents the parameter with the following expression:

$$
k=\frac{2 \Delta \varepsilon_{12}}{\sigma^{y}}
$$

where $\Delta \varepsilon_{12}$ is the deformation of transformation during the austenite-martensite transformations and $\sigma^{y}$ is the yield stress of the austenitic phase. 


\subsubsection{TRIP Evolution versus Volume Proportion ( $Z$ ) of the Martensite Phase}

As seen in other works such as Taleb et al. (2001) [4], estimating the expression in Equation (2) is highly influenced by the applied load during tests. For this reason, we analyze this case with a test using only $75 \%$ of the austenitic phase yield stress during the transformation.

In Figure 20, we present the TRIP evolution of the case presented in Section 3.1.2 vs. the volume proportion determined from the FD test applied just before it. However, as we saw in the previous section, there are expressions such as Desalos' and Leblond's models that allow us to obtain this relation as well. Therefore, we predict the same relation using Desalos' and Leblond's models and compare it to our results.

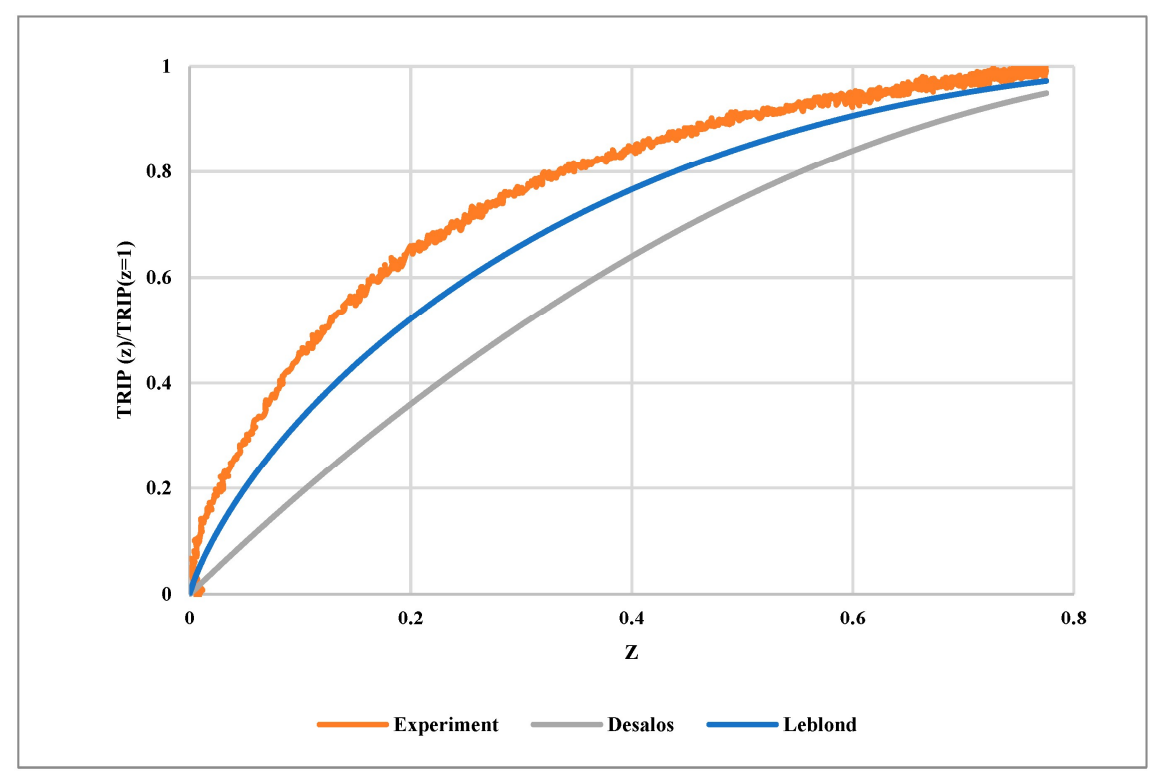

Figure 20. The orange line represents the evolution of the ratio $T R I P(Z) / T R I P(Z=1)$ vs. $Z$ during TRIP with $75 \%$ of the austenitic phase yield stress during the transformation. The grey line represents the prediction using Leblond's model. The blue line represents the prediction using Desalos' model.

There is a clear distinction between the three results, most noticeably how for our experiment's results, there is a much faster TRIP evolution at the beginning of the transformation than the other two. This difference may be shown considering constitutive equations of both models.

Desalos' model is given by

$$
d \varepsilon_{D}^{t p}=k 2(1-z) d z \sigma
$$

If the applied stress and $k$ are assumed constants, Equation (12) can be integrated, leading to

$$
\varepsilon_{D}^{t p}=k z(2-z) \sigma
$$

Therefore,

$$
\frac{\varepsilon_{D}^{t p}(z)}{\varepsilon_{D}^{t p}(z=1)}=z(2-z)
$$

The same reasoning with Leblond's model leads to

$$
d \varepsilon_{L}^{t p}=\frac{2 \Delta \varepsilon_{12}}{\sigma^{y}}(-\ln (z)) d z \sigma
$$


If the applied stress and $\frac{2 \Delta \varepsilon_{12}}{\sigma^{y}}$ are assumed constants, Equation (12) can be integrated, leading to

$$
\varepsilon_{D}^{t p}=\frac{2 \Delta \varepsilon_{12}}{\sigma^{y}} z(1-\ln (z)) \sigma
$$

Therefore,

$$
\frac{\varepsilon_{L}^{t p}(z)}{\varepsilon_{L}^{t p}(z=1)}=z(1-\ln (z))
$$

One can compare Equation (14) and Equation (17):

$$
\frac{\varepsilon_{D}^{t p}(z)}{\varepsilon_{D}^{t p}(z=1)}>\frac{\varepsilon_{L}^{t p}(z)}{\varepsilon_{L}^{t p}(z=1)} \rightarrow z(1-\ln (z))>z(2-z) \rightarrow z>1+\ln (z)
$$

Equation (18) is verified for any $z>0$.

We can conclude that the TRIP kinetics predicted by Leblond's model are more important than the Desalos' kinetics.

\subsubsection{Evaluation of the Linear Relation between the Final TRIP and the Applied Stress}

The relation between the final TRIP and the applied stress is estimated using two approaches: The first one uses only the reference TRIP results (Section 3.1) while in the second one, for each stress level, the average value is considered. The results are expressed in Figure 21.

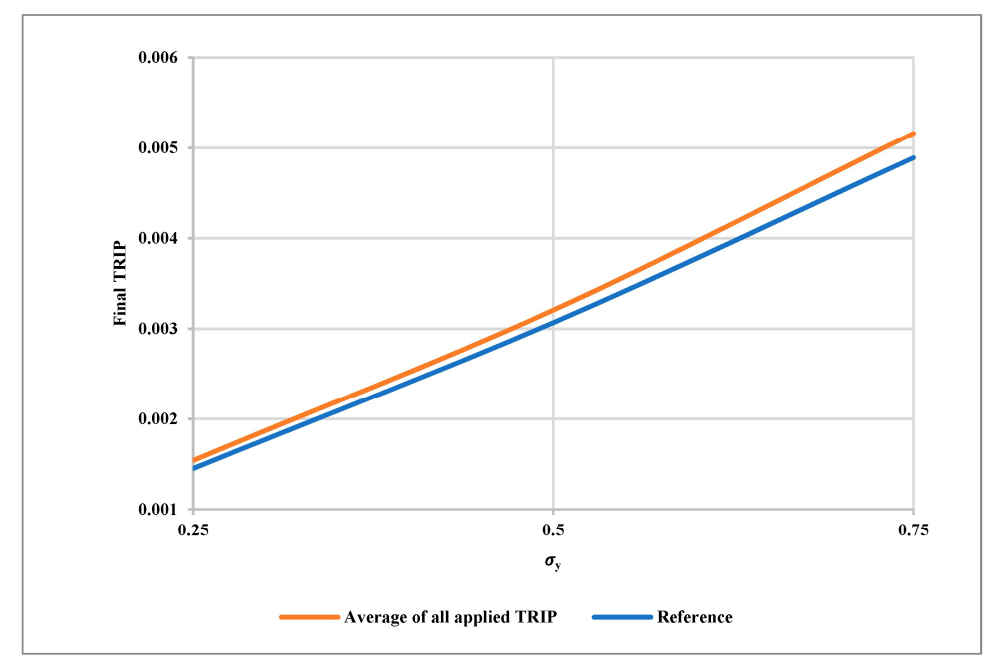

Figure 21. Final TRIP vs. applied stress. In the first case, only the reference TRIP results (found in Section 3.1) are considered. In the second case, for each stress level, the average value is considered.

The results obtained show us a very good signal of how when the elastoplastic histories applied to our test specimens are limited to FD tests and TRIP tests, there is only a small effect noticeable. Note that in the literature, it is generally admitted that the relation is linear for applied stresses that are less than one-half of the yield limit $[4,16]$. Our results in Figure 21 show that such a limit is at least three-quarters of the yield limit.

\section{Concluding Remarks}

In this paper, we have investigated the TRIP phenomenon in the martensitic transformation of 35NCD16 steel, with a main focus on the memory of TRIP after the application of a thermoelastoplastic history including phase changes. In addition, the TRIP parameter, the kinetics of TRIP, as well as the relation between the final TRIP and the applied stress are also studied.

The obtained results enable us to make the following concluding remarks:

- After "light" thermomechanical history composed of only Free Dilatometry, no memory is observed: The TRIP response is completely superimposed on the reference 
test under question. This observation is the same for all stress levels considered here: $\operatorname{TRIP}\left(\sigma_{y} / 4\right), \operatorname{TRIP}\left(\sigma_{y} / 2\right)$, and $\operatorname{TRIP}\left(3 \sigma_{y} / 4\right)$. As a consequence, the specimen becomes reusable even if it is necessary to check for oxidation after a certain number of tests. This "economic" option allows us to perform several "low cost" TRIP tests compared to the classical procedure (one test $=$ one specimen). Finally, note that our result confirms the choice adopted in Coret (2002) [17] and Videau (1996) [18] where they use the same specimen for several TRIP tests.

- After thermomechanical histories including Free Dilatometries as well as TRIP tests, the amount of TRIP is a slightly reduced (by about $10 \%$ ).

- After the elastoplastic cycle, where the austenitic phase undergoes plasticity, the difference is more significant. This result is qualitatively in accordance with the test results given in Taleb (2014) [16] where the interaction between TRIP and Classical plasticity is studied. For a quantitative point of view, the pre-hardening in Taleb (2014) [16] is significantly more important than the one considered in our study, as they reached a plastic strain of the order of $5 \%$. This parameter deserves verification in a future extension of our study.

The study of the TRIP parameter and kinetics points out the importance of Leblond's model with its predictive character.

Author Contributions: Conceptualization, J.J. and L.T.; methodology, L.T.; validation, L.T., formal analysis, L.T.; investigation, J.J. and L.T.; writing—original draft preparation, J.J.; writing-review and editing, J.J. and L.T.; visualization, L.T.; supervision, L.T. All authors have read and agreed to the published version of the manuscript.

Funding: This research received no external funding.

Data Availability Statement: All data has been kept in the National Institute of Applied Science (INSA) of Rouen by one of the authors (J.J.).

Conflicts of Interest: The authors declare no conflict of interest.

\section{References}

1. Taleb, L.; Sidoroff, F. A micromechanical modeling of the Greenwood-Johnson mechanism in transformation induced plasticity. Int. J. Plast. 2003, 19, 1821-1842. [CrossRef]

2. Taleb, L.; Petit, S. New investigations on transformation induced plasticity and its interaction with classical plasticity. Int. J. Plast. 2006, 22, 110-130. [CrossRef]

3. Tahimi, A.; Taleb, L.; Barbe, F. Plasticité induite par transformation de phase martensitique dans l'acier 35NCD16. In Congrès Français de Mécanique; AFM: Courbevoie, France, 2009.

4. Taleb, L.; Cavallo, N.; Waeckel, F. Experimental analysis of transformation plasticity. Int. J. Plast. 2001, 17, 1-20. [CrossRef]

5. Boudiaf, A.; Taleb, L.; Belouchrani, M.A. Experimental analysis of the correlation between martensitic transformation plasticity and the austenitic grain size in steels. Eur. J. Mech.-A/Solids 2011, 30, 326-335. [CrossRef]

6. Greenwood, G.W.; Johnson, R.H. The Deformation of Metals Under Small Stresses During Phase Transformations. Proc. R. Soc. Lond. Ser. A Math. Phys. Sci. 1965, 283, 403-422.

7. Magee, C.L.; Paxton, H. Transformation Kinetics, Microplasticity and Aging of Martensite in FE-31 NI. 1966. Available online: https:/ / www.semanticscholar.org/paper/transformation-kinetics\%2C-microplasticity-and-aging-magee-paxton/12b3 3aea3be1e3e5ad16656b4a40c2986a3156c4 (accessed on 7 September 2021).

8. Otsuka, T.; Satani, D.; Yamamoto, K.; Okamura, K.; Brenner, R.; Bacroix, B. Microstructure and heat treatment effect on transformation strain in steels: Part1 experiment. Mater. Sci. Technol. 2018, 35, 181-186. [CrossRef]

9. Otsuka, T.; Satani, D.; Yamamoto, K.; Okamura, K.; Brenner, R.; Bacroix, B. Microstructure and heat treatment effect on transformation strain in steels: Part 2 modelling. Mater. Sci. Technol. 2018, 35, 187-194. [CrossRef]

10. Simsir, C. Transformation Induced Plasticity (TRIP) of SAE 52100 Steel during Martensitic and Bainitic Transformations. Available online: https://www.researchgate.net/publication/321059412_Transformation_Induced_Plasticity_TRIP_of_SAE_52100_Steel_ during_Martensitic_and_Bainitic_Transformations (accessed on 12 November 2021).

11. Nagayama, K.; Kitajima, Y.; Kigami, S.; Tanaka, K.; Fischer, F.; Cailletaud, G. Transformation Induced Plasticity in Maraging Steel: An Experimental Study. Key Eng. Mater. 2000, 177, 443-448. [CrossRef]

12. Nagayama, K.; Terasaki, T.; Tanaka, K.; Fischer, F.D.; Antretter, T.; Cailletaud, G.; Azzouz, F. Mechanical properties of a Cr-Ni-Mo-Al-Ti maraging steel in the process of martensitic transformation. Mater. Sci. Eng. A 2001, 308, 25-37. [CrossRef] 
13. Nagayama, K.; Terasaki, T.; Goto, S.; Tanaka, K.; Antretter, T.; Fischer, F.D.; Cailletaud, G.; Azzouz, F. Back stress evolution and iso-volume fraction lines in a $\mathrm{Cr}-\mathrm{Ni}-\mathrm{Mo}-\mathrm{Al}-\mathrm{Ti}$ maraging steel in the process of martensitic transformation. Mater. Sci. Eng. A 2002, 336, 30-38. [CrossRef]

14. Zuo, X.; Liu, Y.; Chen, N.; Rong, Y. The process design of water quenching based on finite element simulation and its applications. Heat Treat. Surf. Eng. 2020, 2, 9-15. [CrossRef]

15. Leblond, J.B.; Devaux, J.; Devaux, J.C. Mathematical modelling of transformation plasticity in steels I: Case of ideal-plastic phases. Int. J. Plast. 1989, 5, 551-572. [CrossRef]

16. Taleb, L. Transformation-Induced Plasticity (TRIP). In Encyclopedia of Thermal Stresses; Hetnarski, R.B., Ed.; Springer: Dordrecht, The Netherlands, 2014; pp. 6153-6163. ISBN 978-94-007-2739-7.

17. Coret, M.; Calloch, S.; Combescure, A. Experimental study of the phase transformation plasticity of 16MND5 low carbon steel under multiaxial loading. Int. J. Plast. 2002, 18, 1707-1727. [CrossRef]

18. Videau, J.-C.; Cailletaud, G.; Pineau, A. Experimental Study of the Transformation-Induced Plasticity in a Cr-Ni-Mo-Al-Ti Steel. J. Phys. IV 1996, 6, C1-465. [CrossRef] 